\title{
Kutibu majeraha, kutia matumaini: Ushirikiano wa watanzania dhidi ya fistula obstetrikia
}

Thoraya Ahmed Obaid

Erica Chong

Population Council

Follow this and additional works at: https://knowledgecommons.popcouncil.org/departments_sbsr-pgy

Part of the International Public Health Commons, Maternal and Child Health Commons, Obstetrics and Gynecology Commons, Social and Behavioral Sciences Commons, and the Women's Health Commons How does access to this work benefit you? Let us know!

\section{Recommended Citation}

Obaid, Thoraya Ahmed and Erica Chong. 2005. "Kutibu majeraha, kutia matumaini: Ushirikiano wa watanzania dhidi ya fistula obstetrikia," Quality/Calidad/Qualité no. 16. Dar es Salaam: Utu Mwanamke/ Women's Dignity Project. 
Kutibu Majeraha, Kutia Matumaini:

Ushirikiano wa Watanzania

Dhidi ya Fistula Obstetrikia

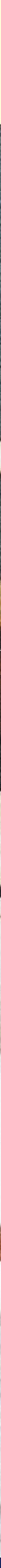

Quality/Calidad/Qualité/ 


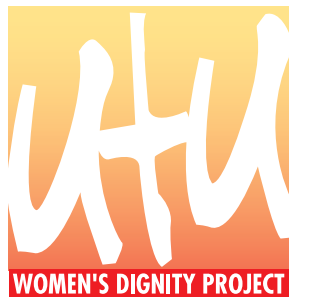

Utu Mwanamke/Women's Dignity Project

S. L. P. 79402, Dar es Salaam, Tanzania

Simu: 022 2152577/8, Nukushi: 0222152986

Barua pepe: info@womensdignity.org

Tovuti: www.womensdignity.org 


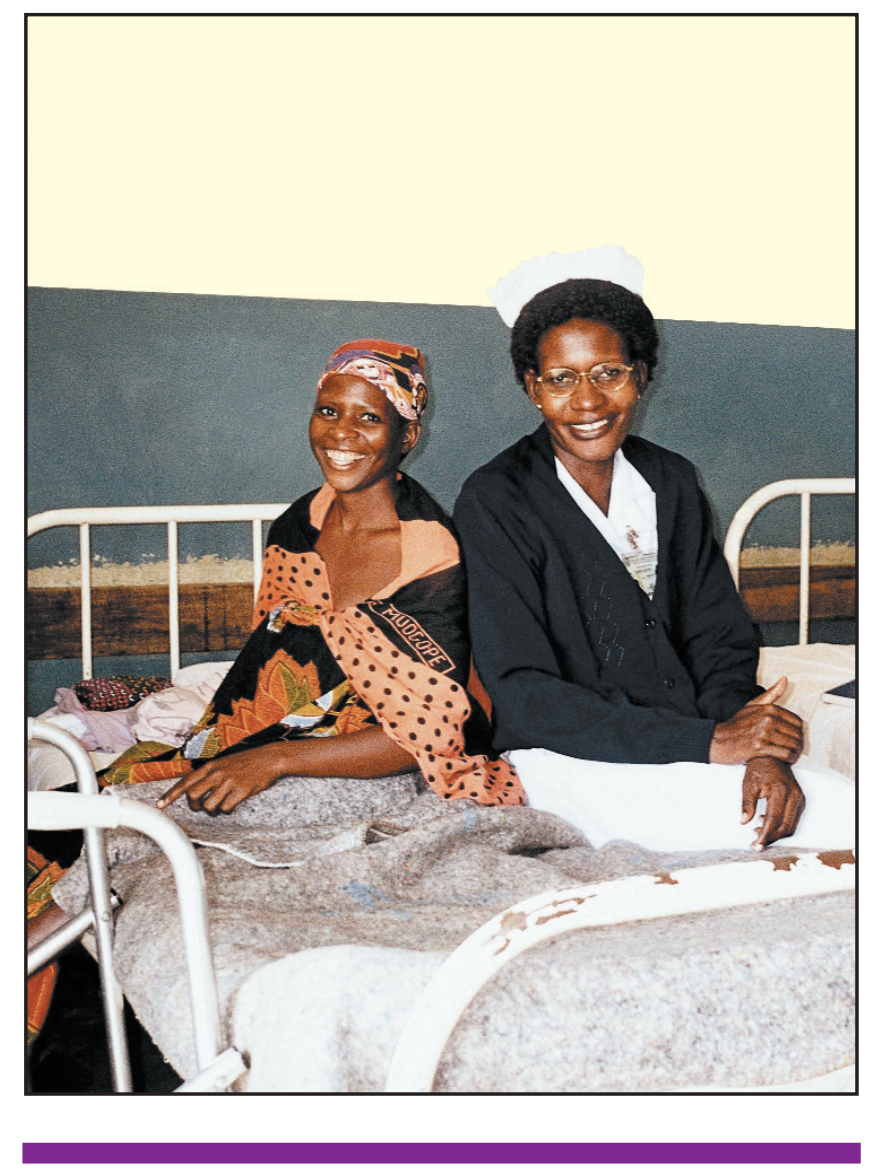

Kutibu Majeraha, Kutia Matumaini: Ushirikiano wa Watanzania Dhidi ya Fistula Obstetrikia 



\section{I B A J I}

Wasichana na wanawake wenye fistula, kila siku wanatukumbusha kwamba lazima tuimarishe huduma ya afya ya mama na mtoto, na kuinua hadhi ya wasichana na wanawake. Fistula inasababishwa na uchungu wa muda mrefu na uzazi pingamizi, ikiwaacha wasichana na wanawake kuvuja mkojo na/au kinyesi mfululizo bila ya kujizuia kwa kupitia njia ya uzazi baada ya kujifungua. Wanaopatwa na fistula wanakabiliana na hali ya unyanyapaa na aibu, na mara nyingine kutengwa na jamii zao. Fistula inazuilika, na imeshatokomezwa katika nchi tajiri. Lazima tuitokomeze fistula nchini Tanzania na vile vile katika nchi nyingine zinazoendelea.

Tanzania iko mstari wa mbele kimataifa katika juhudi za kutatua tatizo la fistula. Tuna hospitali nyingi zinazotoa huduma kwa tatizo la fistula; mpango wa rufaa kusaidia wasichana na wanawake kupata matibabu; Asasi Zisizo za Kiserikali zinazofanya utafiti, kutoa elimu kwa umma na mafunzo juu ya fistula; na ushirika wa Wizara ya Afya, Asasi Zisizo za Kiserikali na wengineo katika kuzuia utokeaji wa fistula kwa ujumla.

Hospitali ya Bugando iliyopo Mwanza imekuwa chombo muhimu kinachoongoza harakati za kutatua tatizo la fistula kwa ufanisi. Wameshirikiana na Wizara ya Afya, Utu Mwanamke na na wadau wengine, kuunda mpango wa kitaifa unaohamasisha usikivu na kuchukua hatua juu ya tatizo la fistula. Tunawapongeza Hospitali ya Bugando kwa juhudi zake, na tunaamini kwamba zitawahamasisha na wengine kulipa uzito suala hili juu ya afya na haki ya wanawake.

Wizara ya Afya imepania kutokomeza fistula, na kuhakikisha wasichana na wanawake wanakuwa na afya na kuishi kwa utu.

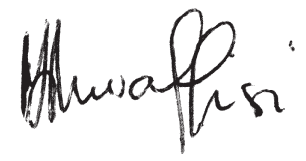

M. J. Mwaffisi

Katibu Mkuu

Wizara ya Afya 
"Qualite," jarida linalochapishwa na Population Council, linadokeza mifano ya mipango ya kikliniki na kielimu inayotoa ahadi nzito na mitazamo vumbuzi na makini kwa suala la huduma bora ya afya ya ujinsia na uzazi. Matoleo haya yamejizatiti katika falsafa kuwa, watu wana haki ya msingi ya kuheshimiwa katika kupewa huduma, kupata taarifa, kuchagua na haki ya kutembelewa na watoa huduma ya afya.

Nyaraka za Miradi ya "Qualite" zimepiga hatua nzuri katika moja au zaidi ya mambo yafuatayo; kupanua wigo wa uchunguzi wa mbinu na teknolojia iliyopo, kutoa taarifa ambazo wateja wanazihitaji kufanyia maamuzi ili kuwawezesha wateja kuwa walezi bora wa afya ya ujinsia na uzazi; kufanya juhudi za uvumbuzi wa kuongeza uwezo wa utawala bora na kupanua stadi za watoa huduma katika ngazi zote; kwa kujumuisha huduma ya afya, uzazi wa mpango na huduma zinazohusiana katika njia ya uvumbuzi, na kuwafikia wale wasiopata huduma kikamilifu na wale waishio katika mazingira magumu.
Miradi inachaguliwa ili kuhifadhiwa na kundi la ushauri linaloundwa na watu wenye ujuzi wa aina mbalimbali katika kuinua ubora wa huduma ya afya ya ujinsia na uzazi. Hakuna mradi hata mmoja uliohifadhiwa unaoweza kutolewa kama mfano wa kuigwa. Kila mradi unawasilishwa kama mfano wa uvumbuzi wa pekee wa maadili, madhumuni na utekelezaji. Uzoefu huu wa kujifunza unaoonyesha mwenendo wa mtu unaotakiwa kujipima kwa mahitaji ya baadaye ya mteja na kutafuta mbinu inayofaa ili kupata mahitaji hayo. Hali hii inayoakisiwa, inakuzwa na kule kukubali mabadiliko katika mahitaji ya mteja na pia kwa mageuzi mapana zaidi ya kijamii na kiuchumi yanayoathiri jamii mbalimbali. Uwekaji nyaraka za chaguo muhimu zilizofanywa na mpango huu, kusaidie kuimarisha kwa vitendo, imani kuwa kuridhika kwa mtu binafsi na huduma za ujinsia na za afya ya uzazi zinazotolewa, kunahusiana sana na mafanikio ya malengo mapana zaidi ya afya na idadi ya watu.

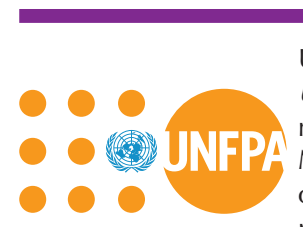

Uchapishaji wa toleo hili la "Qualite" umewezekana kutokana na msaada uliotolewa na United Nations Population Fund (UNFPA); na Programu ya Robert H. Elbert kuhusu masuala muhimu katika Afya ya Uzazi ya Population Council; Ford Foundation; na Mpango wa Jinsia, Familia na Maendeleo ya Population Council. Kauli na maoni yaliyotolewa katika makala haya ni dhima ya waandishi na sio ya shirika lolote linalotoa msaada kwa "Qualite".

\section{(B) Population Council}

Population Council ni Asasi ya Kimataifa isiyo ya kiserikali, isiyotengeneza faida, yenye kutafuta kuboresha maisha na afya ya uzazi ya kizazi cha sasa na kijacho duniani pote na husaidia kufikiwa kwa ubinadamu, usawa na uwiano endelevu kati ya watu na raslimali. Baraza linafanya utafiti wa kimatibabu, sayansi ya jamii na utafiti wa afya ya umma na husaidia kujenga uwezo wa utafiti katika nchi zinazoendelea. Lilianzishwa mwaka 1952, Baraza linasimamiwa na Bodi ya Kimataifa ya Wadhamini. Makao Makuu yake ya New York yanasaidia mtandao wa ofisi zake za kanda na katika kila nchi duniani kote.

Population Council, One Dag Hummarskjold Plaza, New York, NY 10017, Marekani

Simu: (212) 339-0500, Faksi (212) 755-6052, Barua pepe: qcq@popcouncil.org tovuti:http://www.popcouncil.org

Picha ya Jalada na Erica Chong.

Toleo Na. 16 ISSN: $1097-8194$

Hakimiliki (C) 2004 The Population Council, Inc.

Sehemu yoyote ya chapisho hili inaweza kunakiliwa au kuchukuliwa kwa kutimiza mahitaji ya mtumiaji bila idhini toka Population Council, ili mradi tu zile sehemu zilizonakiliwa zinagawiwa bure au kwa gharama (isiyo na faida) na pia chanzo kitambuliwe. Population Council itashukuru kupata nakala ya maandishi yoyote ambapo matini zake zimetumika. 


\title{
U t a n g u 1 i z i
}

\author{
Na Thoraya Ahmed Obaid
}

Katika sehemu nyingi ulimwenguni, wanawake wanajifunza kukubali hatari za magonjwa, majeraha na vifo kama malipo wanayopaswa kulipa kwa kujifungua watoto. Kwa hakika nusu milioni ya wanawake na wasichana wanadhihirisha hili kutokana na vifo vyao kila mwaka. Kati ya wanawake wanaonusurika katika uzazi kila mwaka, wengi wao wanajikuta afya zao sio nzuri. Wanawake hawa wanaugua bila sababu kwani matatizo yote yanayohusiana na uzazi yanazuilika. Viwango hivyo vya vifo vya mama wajawazito vilivyopo karne hii ya ishirini na moja vinaashiria pengo kubwa lililopo kati ya nchi zilizoendelea na nchi zinazoendelea, hususani katika ubora na upatikanaji wa huduma ya afya ya uzazi.

Mfano mahususi na wa kutia uchungu wa tofauti katika huduma ni tatizo la kutokwa na mkojo bila kujiziuia-fistula obstetrikia (tazama kisanduku juu ya Fistula obstetrikia na matatizo yake ya kitiba). Fistula Obstetrikia imetokomea katika nchi tajiri zaidi duniani. Hata hivyo kila mwaka Shirika la Afya Duniani (WHO) limekadiria kuwa wanawake na wasichana kati ya 50,000 hadi 100,000, wanaoishi katika jamii maskini wanapatwa na fistula. Tatizo hilo lipo zaidi katika Bara la Afrika.

\section{Chanzo Chake ni U m a s k i i}

Ingawaje tatizo la kutokwa na mkojo bila kujizuia (Fistula) mara nyingi linasababishwa na uzazi pingamizi, hali hii huanza mapema kabla ya wakati wa kujifungua na chanzo chake ni umaskini.

Kudumaa kunakosababishwa na utapiamlo kunaweza kusababisha nyon- ga kutokua vizuri na hivyo kuongeza hatari ya uzazi pingamizi. Vivyo hivyo, wasichana wanaoolewa wakiwa bado wadogo, mara nyingi kwa sababu za kiuchumi, wanapata mimba kabla ya kukua kwao wenyewe hakujakamilika. Wanawake watu wazima waliodhoofishwa kwa kubeba mimba mara kwa mara wana uwezekano mkubwa wa kupatwa na tatizo hili.

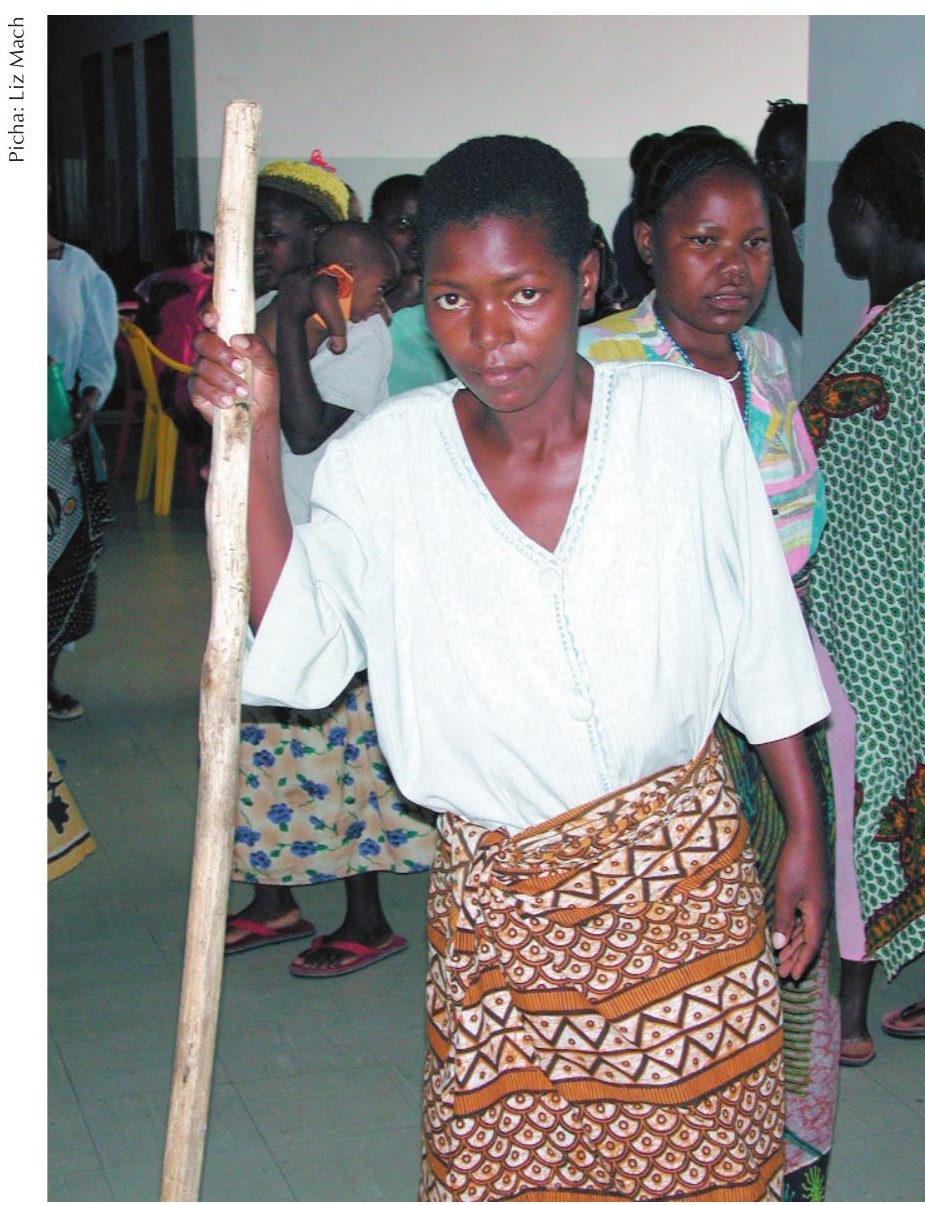

Wanawake wenye fistula obstetrikia wanapata matatizo mengi ikiwa ni pamoja na kushindwa kudhibiti utokaji wa mkojo na/ au kinyesi (ufukunyungu). Mara nyingi wanapatwa na matatizo makubwa kijamii ikiwa ni pamoja na kutokuwepo mfumo wa misaada ya kijamii na kiuchumi, pamoja na kupoteza mtoto wakati wa kujifungua. 
Zaidi ya hivyo, wanawake waliopo katika hali hiyo, mara nyingi wanaishi katika mazingira ambayo afya yao wenyewe hawaipi kipaumbele na huduma zilizopo hazitoshelezi.

Matatizo ya fistula ni makubwa. Wanawake wenye fistula wanapata matatizo mengi ya kitiba ikiwa ni pamoja na kushindwa kudhibiti mkojo na/au

Fistula inaweza kutibiwa kwa upasuaji hata ikiwa miezi au miaka kadhaa baada ya kutokea, na dalili nyingi au zote zinaweza kuondolewa na mama anaweza kurudia hali ya utu wake na kuishi maisha ya kawaida.

kinyesi (ufukunyungu). Kijamii kuna matatizo makubwa zaidi. Kimsingi mwanamke mwenye fistula siyo tu anaelemewa na tatizo la kupoteza mtoto bali pia hapati msaada wa kijamii. Anaweza kukataliwa na mume wake kutokana na kutokwa na mkojo na/au kinyesi bila kujizuia na hali ya uchafu alionayo. Wakati mwingine hata familia yake alikozaliwa wanakataa kumchukua. Anakuwa ametengwa na jamii na anaweza kudhihakiwa au kulaumiwa kwa yaliyomtokea. Pamoja na matatizo hayo, wanawake wanaomudu kuishi na tatizo la fistula huwa wana ujasiri mkubwa na ni imara.

\section{Fistula Si Kuteseka Maisha Yote}

Fistula inaweza kutibiwa kwa upasuaji, hata ikiwa miezi au miaka kadhaa baada ya kutokea na dalili nyingi au zote zinaweza kuondolewa na mama anaweza kurudia utu wake na kuishi maisha ya kawaida. Kwa kulinganisha na mahitaji, huduma za matibabu ya fistula hazitoshi na wataalamu wa upasuaji ni wachache. Nchi nyingi zinajitahidi kuboresha na kuongeza huduma ya afya katika muktadha wa ushindani mkali kwa raslimali ndogo (hususani wataalamu wa upasuaji). Katika mazingira hayo, kumhudumia mwanamke aliyeishi na fistula kwa miaka mingi hakupewi kipaumbele. Zaidi ya hayo, wasichana wengi na wanawake wenye fistula hawafahamu kuwa wanaweza kutibiwa na pia hawana uwezo wa kulipia matibabu au hata gharama za usafiri wa kuwafikisha kwenye vituo vya upasuaji.

\section{F i s t u I I n a z u I i k a}

Ili kuzuia fistula, upatikanaji wa huduma ya afya ni muhimu. Kunahitajika mchanganyiko wa huduma bora kabla ya kujifungua ikiwa ni pamoja na uchunguzi, kuwepo na wakunga wenye ujuzi wakati wa kujifungua, na kutolewa kwa matibabu ya haraka inapotokea dharura na huduma bora baada ya kujifungua, yote haya yanaweza kuokoa maisha ya kina mama na kuzuia ulemavu wa aina nyingi ikiwa ni pamoja na fistula obstetrikia. Kuondoa fistula obstetrikia ni suala linalovuka mawanda ya huduma ya afya pekee. Kunahitajika mtazamo wa pamoja, unaojumuisha msukumo na kujidhatiti kwa nchi husika na jumuiya ya kimataifa. Kunahitajika pia ushirikiano wa hali ya juu toka jumuiya mbalimbali katika kuboresha uzuiaji na matibabu ya fistula. Juhudi za elimu ni muhimu ili kukomesha unyanyapaa na kuhakikisha kuwa ufahamu wa mikakati ya uzuiaji unafikia familia na jamii nzima. Mkakati wa pamoja wa uzuiaji ni lazima uhusishe juhudi za kuimarisha usawa wa wanawake, katika masuala ya upatikanaji wa huduma ya afya, elimu, uzalishaji, mapato na uhuru wa kufanya maamuzi. Mitazamo makini ya uboreshaji wa huduma na kuhimiza mabadiliko ni muhimu sana katika maeneo ya vijijini na yaliyo mbali na mijini. Wanawake waliofanikiwa kupata matibabu wanahitaji pia msaada wa kijamii ili kuwasaidia kurudi katika jamii zao. 


\section{Tanzania: Mfano wa Juhudi za $\mathrm{K}$ it a if a}

Mfano ufuatao unaeleza juhudi zilizopo Tanzania za kutokomeza fistula obstetrikia. Viongozi katika Hospitali yenye mpango, Asasi Zisizo za Kiserikali (AZISE), na serikali ya Tanzania, wanafanya juhudi za pamoja katika kuwabainisha na kuwatibu wanawake wenye tatizo la fistula, wakati huo huo wakibainisha na kupambana na sababu za tatizo hili. Uchunguzi kifani unaelezea visa vya wanawake na wasichana wanaofika katika Hospitali ya Bugando, na jinsi watumishi wa hospitali wanavyojitolea kwa dhati. Pia unaelezea wanaharakati katika jamii na viongozi wa serikali, wanaoelewa umuhimu wa kutibu na kuzuia fistula na wale wanatoa misaada kwa ajili ya mahitaji hayo.

\section{UNFPA na Washirika Wake - Jitihada za Kiulimwengu}

Mwaka 2003, Mfuko wa Shirika la Umoja wa Mataifa la Idadi ya Watu (UNFPA), lilitambua kuwa fistula ni tatizo la afya ya wanawake, lakini halijapewa umuhimu kwa muda mrefu. Mfuko ulizindua kampeni ya dunia ya kutokomeza fistula. Kwa kushirikiana na mashirika ya Umoja wa Mataifa, serikali, asasi zisizo za kiserikali, asasi za tiba na wataalamu binafsi, lengo la kampeni lilikuwa kuongeza ufahamu, upatikanaji wa mahitaji, kupanua matibabu na kushughulikia chanzo cha fistula. Tafiti za hivi karibuni zilizofanywa Bangladesh na Yemen zimeonyesha kuwa fistula obstetrikia ni tatizo linalogusa nchi nyingine pia, nje ya Afrika. Hivyo kampeni imeitikia kwa kupanua wigo wa juhudi zake na kuzifikia kanda hizo. UNFPA na washirika wake imekamilisha uainishaji wa mahitaji kwa nchi 14, na wanakusudia kukamilisha uainishaji kwa nchi zote zilizo katika hatari zaidi kufikia mwaka 2005. Kwa hivi sasa, UNFPA inatoa msaada kwa nchi zaidi ya 20, nyingi zikiwa Afrika chini ya Jangwa la Sahara na Asia ya Kusini. Katika kanda zote, UNFPA, pamoja na washirika wake,

Kuzuia na kutibu fistula obstetrikia siyo tu suala la mazoea ya kitiba; bali ni suala la haki za binadamu, ikiwa ni pamoja na haki ya kupata huduma ya afya ya uzazi inayotosheleza

inaandaa mikakati ya kitaifa ya kupambana na mapengo yaliyobainishwa katika uzuiaji na tiba ya fistula obsterikia na kusaidia kutekeleza mipango hiyo.

Ufumbuzi wa vitendo usielemewe na ukubwa na ugumu wa tatizo. Kila upasuaji wa fistula unapofanyika unafunua maisha mapya kwa mwanamke mmoja. Kila mafanikio ya upasuaji yanaonyesha jamii kuwa, fistula siyo kifungo cha maisha na hivyo kuongeza kuaminiwa kwa huduma ya afya. Kila mkunga aliyepata mafunzo na kila huduma inayoboreshwa ili kutoa matibabu bora na ya haraka kwa tatizo la uzazi, inachangia katika uzuiaji wa hali hii. Kila juhudi za kielimu zinazofanywa katika jamii zinaweza kuongeza ufahamu wa familia nyingi kuhusu umuhimu wa kuhakikisha kuwa wanawake wanapata huduma wanayoihitaji.

Kuzuia na kutibu fistula obstetrikia siyo tu suala la mazoea ya matibabu, pia ni suala la haki za binadamu ikiwa ni pamoja na haki ya kupata huduma ya afya ya uzazi inayotosheleza. Wanawake na wasichana duniani kote wanastahili kupata muda, raslimali na kujitolea ili kufikia lengo hili. Wakati ndiyo umefika wa kuanza kwa ufanisi ili kurudisha matumaini na utu kwa kila mwanamke anayejifungua. 


\section{Fistula Obstetrikia ni nini?}

Fistula obsterikia ina maana ya tundu linalotokea wakati mwanamke anapojifungua katika misuli kati ya uke na kibofu cha mkojo au kati ya uke na njia ya haja kubwa au vyote viwili. Katika nchi zinazoendelea sababu ya fistula ni kutokana na uzazi pingamizi unaohusiana na hali ambayo nyonga ya mama ni ndogo na hivyo hushindwa kuruhusu kichwa cha mtoto kupita salama. Katika mazingira yenye kuwa na huduma za dharura za uzazi, hususani watoaji wa huduma za upasuaji wa kutoa mtoto, wanaweza kudhibiti matukio mengi ya uzazi pingamizi. Wakati ambapo huduma ya upasuaji wa kumtoa mtoto haipatikani au haitolewi kwa wakati unaofaa, msukumo wa kichwa cha mtoto unaweza kusababisha misuli ya uke ishindwe kufanya kazi na hivyo kujiachia na matokeo yake ni mama kupata fistula, au tundu kwenye misuli. Karibu kwa kila tukio la fistula, uzazi pingamizi pia unasababisha kifo cha mtoto aliyeko bado tumboni.

Wanawake wenye fistula wanaweza kuugua magonjwa kadhaa ikiwa ni pamoja na kushindwa kudhibiti utokaji wa mkojo na/au kinyesi (ufukunyungu), maambukizo ya kibofu cha mkojo, vidonda kwenye uke. Pia kati ya wanawake watano, mmoja anaugua ugonjwa wa "ulemavu wa kanyagio" unaosababisha kuharibika kwa mishipa katika mguu mmoja au yote na kusababisha ulemavu wa kutembea. (UNFPA na EngenderHealth 2003). Kwa wanawake wengi mzunguko wao wa hedhi huathirika na wengine wanapoteza uwezo wao wa kushika mimba.

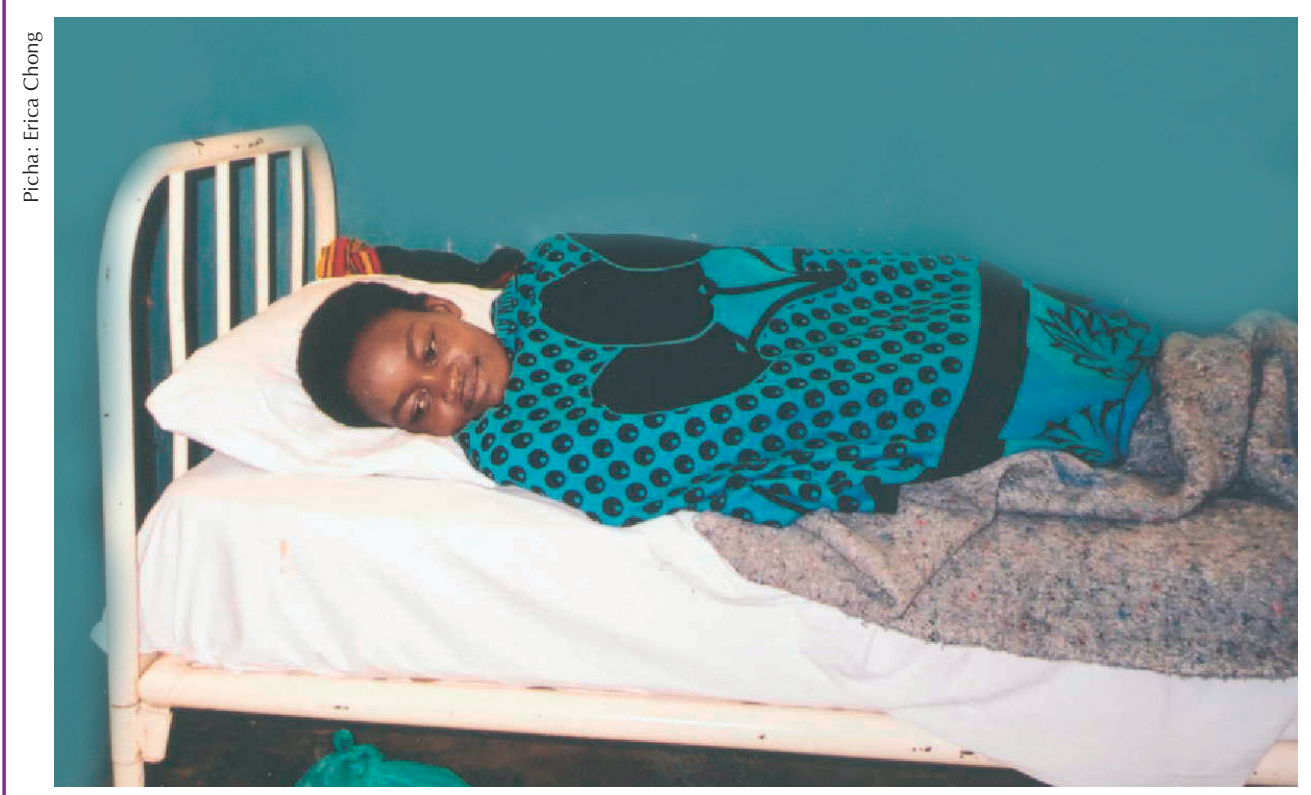

\section{Mar e j e o}

United Nations Population Fund (UNPF) na EngenderHealth, 2003. Fistula Obstetrikia Taarifa ya Tathmini ya Mahitaji: Matokeo toka nchi tisa za Kiafrika. New York, Umoja wa Mataifa. 


\section{Kutibu Majeraha, Kutia Matumaini:}

Ushirikiano wa Watanzania

\section{Dhidi ya Fistula Obstetrikia}

Na Erica Chong

Msichana alikuja hospitali akiwa na fistula ya haja kubwa na pia kati ya kibofu na uke. Daktari alipomchunguza alikuta tundu moja kubwa; njia ya mkojo, uke na njia ya haja kubwa, vyote viliungana na kuwa tundu moja kubwa. Mtaalamu wa upasuaji aliyekuwa anatembelea hospitali hiyo, alitumia muda wa saa moja na nusu kurekebisha kibofu cha mkojo na kurekebisha njia ya mkojo. Alipumzika, alijitikisa kuondoa mavune katika mabega yake akashauriana na wataalamu wengine wa upasuaji, na kuendelea kumhudumia mgonjwa kwa muda wa saa moja na nusu nyingine, alimudu kurekebisha njia ya haja kubwa na misuli banifu ya njia ya haja kubwa. Wakati akianza kurekebisha uke, ujuzi wa mtaalamu huyu ulienea kwenye vyumba vya upasuaji, baadhi ya wataalamu wa upasuaji walifika katika chumba cha upasuaji kuangalia upasuaji unaofanywa na mtaalamu huyu. Wakati mtaalamu huyu akiendelea kumhudumia mgonjwa ukimya ulitawala, lakini alipomaliza furaha na msisimko ulienea kote, baadhi ya watu walipiga makofi, wengi walicheka, wakiwa hawaamini kile walichokiona. [Daktari mmoja] alianza kuimba nyimbo za kumtukuza Mungu-ilikuwa hali ya kupendeza kwani watu wengine waliimba pia katika sauti iliyojaza chumba. Nilianza kuelewa ukubwa wa jambo tunaloweza kukamilisha hapa, kimsingi jinsi tunavyoweza kuboresha maisha ya hawa wanawake.

- Liz Mach, Hospitali ya Bugando

Tanzania ni nchi katika Afrika Mashariki yenye uzuri mkubwa, inajulikana sana nje kwa utajiri wake wa wanyama pori katika mbuga kama za Serengeti na Ngorongoro. Ingawa Kiswahili na Kiingereza ndizo lugha rasmi nchini, Tanzania ina watu milioni 35 toka makabila zaidi ya mia moja, kila moja ikiwa na lugha na desturi zake.

Pamoja na utajiri wake wa asili, Tanzania inajitahidi kupambana na changamoto ya maendeleo; karibu asilimia 60 ya idadi ya watu wanaishi chini ya dola 2 za Kimarekani kwa siku (UNFPA 2003) na umri wa kuishi umepungua hadi miaka 43 (Benki ya Dunia 2003). Matumizi ya serikali ni dola 6 za Kimarekani kwa kila mtu, na nchi ina madaktari wanne kwa kila watu 100,000 (ikilinganishwa kwa mfano na Namibia ambayo ina madaktari 29 kwa kila watu 100,000 (UNFPA 2003). Mfumo imara wa elimu uliokuwepo
Tanzania umeanza kudidimia miaka ya hivi karibuni. Ingawaje kufutwa kwa ada ya shule za msingi kumeongeza uandikishaji wa wanafunzi, gharama zinazohusiana na vitabu, sare na malipo mengine yasiyo rasmi, vyote hivi vinazuia baadhi ya watoto kuandikishwa shuleni. Kiasi cha asilimia 5 ya watoto wenye umri wa kuingia shule za sekondari ndiyo wanapata fursa hiyo (PRB 2000).

Wasichana wanaondolewa shuleni mapema zaidi ya wavulana, na hivyo kuachwa bila stadi za kutosha za kuwasaidia kupata mapato katika maisha yao, hii inaongeza uwezekano wa kujihusisha katika tabia hatarishi ya ngono ili kupata fedha za kujikimu. Umaskini unasababisha wazazi kuwaozesha mabinti zao mapema ili kuepuka gharama za kuwatunza. Ingawa katika Tanzania wastani wa umri wa kuolewa ni miaka 18.3 (PRB 2000), umri wa chini kisheria ni miaka 15 kwa wasichana na miaka 18 kwa wavulana, 
na hivyo ni jambo la kawaida kwa wasichana kuolewa wakiwa na miaka 15 au 16.

Katika jamii inayothamini wanawake kwa sababu ya uwezo wao wa kuzaa, wasichana wanashinikizwa kushika mimba haraka iwezekanavyo baada ya kuolewa; Kutumia uzazi wa mpango kabla ya uzazi wa kwanza si jambo linalokubalika kwa urahisi. Asilimia 39 ya wasichana wenye umri wa miaka 18 , tayari wana watoto au wana mimba za watoto wao wa kwanza (PRB 2001). Bahati mbaya, mimba nyingi za mapema zinatokana na matokeo ya umaskini.

Kwa sababu kufanya utaratibu wa kujifungulia hospitali ni vigumu, wanawake wengi wanajifungulia nyumbani. Wakati mtoto wa Rhobi aliposhindwa kutoka, alitembea au kukaa kwenye baiskeli umbali wa kilometa 22 ambapo watumishi wa Kliniki walimpeleka kwa gari hadi Hospitali. Alipofanyiwa upasuaji mtoto wake alikuwa tayari amefariki na Rhobi akapata fistula

Utoaji mimba usio salama na kuzaa kunasababisha vifo kati ya 7,500 hadi 15,000 kwa wasichana na wanawake wa Tanzania kila mwaka, zaidi ya hayo wanawake 150,000 hadi 450,000 hupata ulemavu unaotokana na matatizo yanayohusiana na mimba (TPP 2003)1.

Moja ya matatizo makubwa ya kujifungua ni tatizo la fistula obstetrikia. Kama ilivyoelezwa katika kisanduku katika ukurasa wa 4, fistula inabainishwa kwa kutoa mkojo na/au kinyesi mfululizo na pia huambatana na kuzaa mtoto mfu. Wasichana na wanawake wenye fistula pia wanaweza kupata ulemavu wa miguu kutokana na uharibifu wa mishipa, hii hufanya wapate matatizo ya kutembea au kushindwa kabisa kutembea.

Nchini Tanzania, siyo rahisi kukadiria ukubwa wa tatizo la fistula. Takwimu za hospitali zinaonyesha idadi ya chini kwa sababu matukio mengi yanawapata wasichana na wanawake ambao hawafiki hospitali. Takwimu za hospitali zinazohusu upasuaji wa fistula zinaonyesha idadi ndogo ya wagonjwa wanaofahamu kuwa fistula inaweza kutibika na wenye uwezo wa kumudu kutafuta fedha kwa ajili ya kupatiwa matibabu. Siyo rahisi kukusanya takwimu za utafiti kwa sababu ya unyanyapaa unaohusishwa na hali hiyo; juhudi zinazohusika katika kuwafikia wagonjwa wa fistula wanaoishi maeneo ya vijijini, ambapo wagonjwa wengi ndiko walipo si kubwa, na kwa sababu maskini wa Tanzania hawana sauti. Inakadiriwa kuwa matukio mapya 1,200 ya fistula ya obstetrikia hutokea kila mwaka Tanzania. Mwaka 2000-01 karibu wasichana na wanawake 700 walipatiwa matibabu ya upasuaji wa kurekebisha hali hiyo. Hii ikawabakisha wanawake 500 katika mwaka mmoja tu (WDP 2002). Ukizidisha idadi hiyo kwa miongo ambayo wanawake hawakupata matibabu, waliosalia ni wengi.

\section{S a b a b u z a F i s t u I a}

Katika Tanzania, umaskini ndiyo chanzo cha matokeo mabaya ya afya ya mama wajawazito na uzazi kwa ujumla ambayo husababisha kuenea kwa tatizo la fistula. Utapiamlo na mimba za mapema zinachangia kila moja kipekee katika kuifanya nyonga izuie mtoto kutoka. Utapiamlo unaanza mapema na una athari za muda mrefu, kwa watoto wenye umri wa chini ya miaka mitano. Karibu theluthi moja ya watoto wana uzito mdogo, na karibu nusu yao ni wafupi kuliko wastani wa urefu kwa umri wao (UNDP 2003). Ingawa kuzaa mapema siyo kitu cha kawaida katika Tanzania kama ilivyo katika nchi nyingine zenye kiwango cha juu cha fistula (Kwa mfano Ethiopia na Nigeria) zaidi ya msichana

\footnotetext{
1 Utoaji mimba ni kosa la Jinai nchini Tanzania ila tu hufanywa kwa kuokoa maisha ya mama. Kwa hiyo basi utoaji mimba unafanywa kwa kificho na mara nyingi kukiwa na matokeo mabaya.
} 


\section{Vizuizi vya Upatikanaji wa Huduma}

Wanawake wanachelewa au wanakwepa kwenda hospitali kwa sababu kadhaa. Sababu kubwa zaidi ni ukosefu wa fedha kwa ajili ya gharama za huduma ya afya na usafiri, umbali mrefu kwenda kwenye huduma ya afya, ukosefu wa uwezo wa kufanya maamuzi, na kutoelewa matatizo yanayoweza kutokea wakati wa kujifungua.

Ndebele alikuwa na umri wa miaka 19 alipopata fistula. ${ }^{2}$ Alikuwa mgonjwa mara kwa mara wakati wa ujauzito na alitaka kujifungulia katika hospitali, lakini mama mkwe wake alikataa asifanye hivyo. Mimba ilipotimia miezi saba, Ndebele alipata uchungu na alijifungua mtoto mfu nyumbani. Tatizo la fistula lilianza wakati wa uchungu uliochukua muda mrefu, na siku mbili baadaye alianza kutokwa na mkojo bila kujizuia.

Rhobi alikuwa amejifungua watoto wafu mara tatu alipofikia umri wa miaka 19, na uchungu wake wa mwisho ulimsababishia fistula. Alikuwa akiishi vijijini katika upande wa Magharibi mwa Tanzania, mbali na huduma ya afya. Baada ya kupambana na matatizo wakati akijifungulia nyumbani, alitembea na kupandishwa kwenye baiskeli mwendo wa kilometa 22 hadi kituo cha Afya cha karibu. Watumishi walishindwa kumsaidia, hivyo walimpandisha nyuma ya gari aina ya jeep na kumpeleka umbali wa kilometa 25 kwenye hospitali ya karibu ambako madaktari walimtoa mtoto kwa njia ya upasuaji. Rhobi alikuwa kwenye uchungu kwa siku kadhaa, hata hivyo mtoto alikwa tayari amefariki. Alipata fistula lakini hapakuwa na mtaalamu katika hospitali hiyo aliyefahamu namna ya kutibu fistula, hivyo alikaa kwenye wodi kwa miezi kadhaa.

Mwasiti alikuwa na miaka 22 alipopata mimba. Rafiki yake mvulana alitaka kumwoa, lakini baba yake alikataa asiolewe na kijana huyo. Wakati wa ujauzito, Mwasiti alihudhuria kliniki ya mama wajawazito mara tano, ambako alipimwa na kupewa vidonge vya malaria na vidonge vya madini ya chuma. Wazazi wake waliamua ajifungulie nyumbani kwa sababu gharama za hospitali zilikuwa kubwa na ni mbali sana. Baada ya kuwa kwenye uchungu kwa siku tatu Mwasiti alitembea umbali wa kilometa 10 hadi hospitali ya serikali. Alipofika daktari hakuwepo na wakunga hawakufahamu namna ya kumsaidia. Wakati daktari alipoweza kumwona na kumfanyia upasuaji, mtoto wake alikuwa tayari amefariki. Baada ya siku kadhaa alianza kutoa mkojo bila ya kujizuia.

Bahati alikuwa na miaka 28 alipojifungua mtoto wake wa tano. Alipata mimba ya kwanza alipokuwa na miaka 21, wakati huo alikwishaolewa na aliondoka kwenda kuishi na wakwe zake. Mimba zote za nyuma alijifungulia nyumbani, kwa hivyo aliona hakuna sababu mtoto wake wa tano kujifungulia hospitali. Alipohudhuria kliniki ya waja wazito kwa mara ya nne alipewa chanjo ya pepopunda na vidonge vya madini ya chuma. Baada ya kuwa na uchungu kwa siku mbili, mkunga wa jadi aliyekuwa anamsaidia alimwambia aende hospitali. Wakati Bahati anakwenda hospitali ambako mtoto alitolewa kwa vifaa maalum, mtoto wake alikuwa amefariki. Bahati alianza kutokwa na mkojo akiwa bado yuko hospitali lakini hakuwekewa mpira wala hakuna aliyemweleza nini kimetokea.

\footnotetext{
2Majina yanayotolewa katika mifano ni ya kubuni ili kulinda faragha binafsi.
} 
mmoja Mtanzania kati ya wanne anazaa kabla ya kufikia umri wa miaka 18 .

Matatizo ya usafiri yanazuia wanawake wengi kujifungulia kwenye vituo vya huduma ya afya. Asilimia 78 ya idadi ya watu wanaishi vijijini (UNDP 2003), na familia chache zina gari au zinaweza kumudu kupata usafiri kwa urahisi. Zaidi ya hayo, wasichana na wanawake hawana kauli ya kutoa maamuzi kuhusiana na matunzo ya afya zao. Kimsingi, mama zao, mama wakwe zao na waume zao ndiyo wenye udhibiti wa masuala hayo. Hata kunapokuwa na matatizo ya kujifungua mwanamke anaweza akapoteza muda wake wa thamani kwa sababu tu ni lazima amsubiri mume wake arudi nyumbani toka kazini kupata kibali chake kuomba msaada au kumwomba alipie usafiri wa kwenda hospitali. Ingawa wanawake wengi wanamudu kupata huduma kabla ya kujifungua (Kwa wastani wanafika kwenye huduma mara nne), kufanya mipango ya usafiri kwenda kujifungua ni suala gumu zaidi. Umbali unaweza kuwa mkubwa na tarehe haiwezi kukisiwa ili kupanga mambo kabla.

\section{Ku ish i n Fistu I a}

Kumbukumbu za Ndebele baada ya kipindi cha uchungu ziko wazi. Anaamini kuwa mama mkwe wake ni mchawi na hivyo alimroga. Wazazi wake walimchukua na kumrudisha nyumbani ili kumhudumia na kumtibia vizuri, mbali na tatizo lake la kuendelea kutoa mkojo bila kujizuia. Marafiki na majirani walimtembelea mara kwa mara na wengine walilia walipomwona na hali hiyo. Ndebele amemchukia mume wake kwa kutomtetea kwa mama yake alipokuwa na ujauzito, na kwa kutomtembelea kwa wazazi wake. Alisikia kuwa ameoa mwanamke mwingine.

Mama na dada yake Mwasiti walimpa msaada mkubwa baada ya kurudi nyumbani toka hospitali. Wengine hawakumsaidia. Mjomba wake na wifi yake walimdhihaki na jirani zake walimtenga. Rafiki yake mvulana alimsaidia alipokuwa mjamzito, lakini aliacha kuwasiliana naye baada ya kupata fistula. Kutokana na kupooza kidogo katika mguu mmoja kulikosababishwa na kuharibika kwa mishipa wakati wa uchungu uliochukua muda mrefu, Mwasiti alipata taabu katika kutembea. Hawezi tena kusaidia kazi za shamba na aliacha kuhudhuria kanisani.

Akiwa bado anahuzunishwa kwa kumkosa mtoto wake na kujirekebisha kwa kuishi na fistula, Bahati alidhalilishwa na majirani waliomlaumu kwa kumpoteza mtoto kwa kudai kuwa alimuua mtoto wake kwa kuogopa kujifungua. (Kama ilivyothibitishwa na uchungu uliochukua muda mrefu na dai lake la kutaka kwenda hospitali). Mara tu aliporudi nyumbani, mume wake akawa mgonjwa. Alipelekwa hospitali, wiki mbili baadaye alifariki. Bahati aliendelea kuishi na wakwe zake lakini walimfanyia visa vingi. Walimnyima chakula na waliacha kuzungumza naye kabisa. Baada ya miezi mitatu ya kutendewa hivyo Bahati aliwachukua watoto wake na kurudi nyumbani kwa wazazi wake. Kule hawakumyanyapaa. Familia yake ilimhurumia kutokana na mikosi hiyo, na majirani zake wa zamani walikuja kumtembelea. Utokwaji wa mkojo bila kujizuia uliongezeka, na hivyo hakuweza kusafiri mbali na maeneo ya nyumbani kwao, na alikuwa anahofia harufu mbaya inayosababishwa na hali hiyo. Bahati alifadhaishwa sana kuhusu kifo cha mumewe na mtoto wake na pia kuhusu tatizo lake la fistula. Alifikiria sana jinsi atakavyoweza kujisaidia mwenyewe. 
Kama ilivyofafanuliwa hapo juu, fistula ina matokeo mabaya kwa wasichana na wanawake. Kuendelea kutokwa na mkojo na/au kinyesi kunasababisha harufu mbaya na inachangia kusababisha michubuko kwenye uke na maambukizi ya mara kwa mara. Pia baadhi ya wanawake wanapata ulemavu na kushindwa kutembea, wengine wanashindwa kushika mimba tena. Wanawake wa Tanzania wenye fistula pia wanakuwa na matatizo ya kimhemko na kiuchumi yanayoambatana na fistula; hususani inayotokana na kupoteza mtoto, aibu, dhihaka, kutengwa ingawa siyo mara nyingi lakini pia kutelekezwa na wanaume, familia, marafiki na wanajamii. Wengi wanakuwa hawana uwezo wa kuendelea kufanya kazi, na wengi wanaona aibu (au hawaruhusiwi) kushiriki katika shughuli mbalimbali za maisha ya jamii kama vile kushiriki katika ibada za dini na katika sherehe. Hata hivyo, wanawake wa Tanzania, kama wanawake wa sehemu nyingine, pia huonyesha kuwa wao ni amana na wana ushupavu wa kujikimu wenyewe na familia zao pamoja na kutafuta huduma za matibabu.

Kwa miaka mingi, wataalamu wa afya katika Tanzania wamekuwa wakijihusisha na wagonjwa wa fistula lakini hawakufahamu namna ya kulikabili tatizo hili lililoenea nchi nzima. Katika miaka ya kati ya 1990, mfululizo wa matukio uliwakutanisha watu mbalimbali ambao walikuwa na azma moja ya kushughulikia tatizo hili ambalo limetelekezwa kwa muda mrefu. Walianzisha mradi wa fistula katika Hospitali ya Bugando. Baadhi ya waanzilishi wa mradi walipata majukumu mapya kwenye sekta ya raia au serikalini na wameendelea kushughulikia tatizo la fistula. Toleo hili la "Qualite" linawasilisha uzoefu wa Hospitali ya Bugando na ushirika wake wa sasa na mradi wa Utu Mwanamke (Asasi isiyo ya kiserikali) na Wizara ya Afya ya Serikali ya Tanzania, katika kuboresha na kupanua huduma za matibabu ya fistula, njia za kuzuia, harakati za utetezi na kutoa mafunzo katika ngazi za jamii, asasi, taifa na kimataifa.

\section{Kuanzia Mwanzo}

\section{Mkutano wa Ugunduzi}

Dk. Baltazar Gumodoka, Mkurugenzi wa Mradi wa Fistula ameanza kazi ya udaktari wa tiba tangu mwaka 1975. Alianza kuguswa na tatizo la fistula alipokuwa kwenye mafunzo yake ya uzazi /magonjwa ya wanawake katika hospitali ya Muhimbili Dar es Salaam, ambapo mmoja wa maprofesa wake alionesha namna ya kurekebisha tatizo la fistula. Alipopelekwa hospitali moja ya Mkoa wa Mara mwaka 1982, Dk. Gumodoka alijaribu kutumia utaalamu aliojifunza Muhimbili. Hata hivyo hospitali hiyo haikuwa na vifaa vya kutosha vya kuweza kuwahudumia wagonjwa wa fistula waliolazwa.

Baada ya miaka mitano, Dk. Gumodoka alihamishiwa katika hospitali ya Bugando iliyoko Mwanza. Bugando ni hospitali ya pili ya rufaa nchini Tanzania, ikiwa na vitanda 850 na wafanyakazi 800. Hospitali hii inatoa huduma katika eneo lenye watu karibu milioni 10. Akiwa amekuta vifaa bora zaidi, Dk. Gumodoka aliamua tena kutoa huduma za fistula. Alifanya upasuaji wa wagonjwa 20 wa fistula. kwa mwaka, lakini kwa sababu hakuweza kuongeza ujuzi na stadi za kisasa, na hospitali haikuwa na mpango wa mafunzo kwa wauguzi wake, juhudi zake hazikufanikiwa kwa asilimia 50 .

Wakati Dk. Gumodoka anaendelea na kukata tamaa, mtaalamu wa afya toka Marekani, kwenye mazingira tofauti kabisa alikuwa na ari ya kusaidia tatizo hili. Maggie Bangser alikuwa anafanya kazi Asia kwenye shirika la International Women's Health Coalition. Alisikia kwa mara ya kwanza kuhusu fistula, toka kwa mwenzake aliyekuwa anafadhili mpango wa matibabu ya fistula nchini Nigeria. Maggie alihamishiwa Afrika Mashariki 


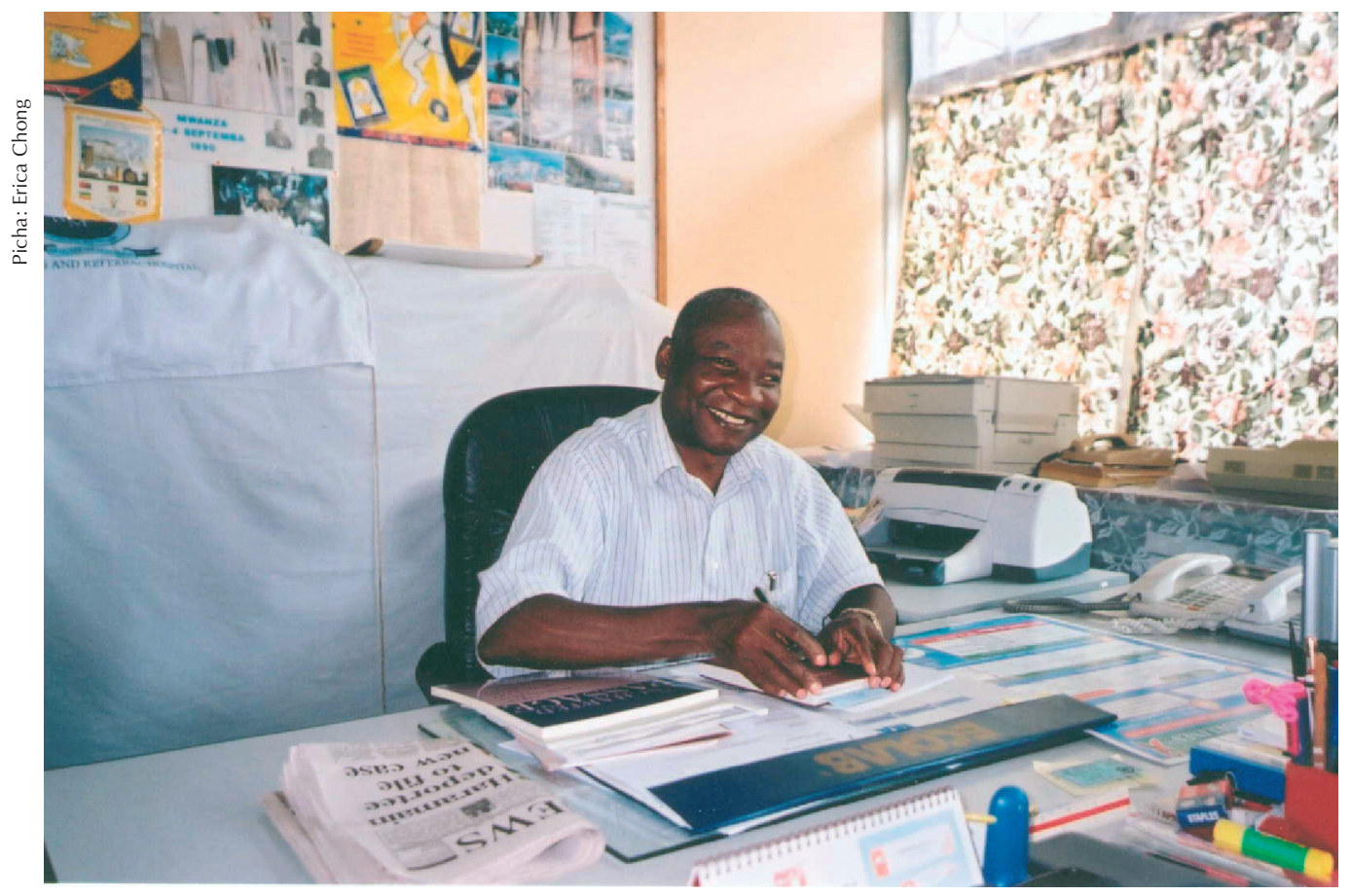

Wakati wa mafunzo yake ya udaktari, Dk. Gumodoka aliona matatizo ya kukera ya fistula. Alijijengea ari ya kutaka kuwasaidia wagonjwa hawa, lakini upungufu wa vifaa na mafunzo vilimkwamisha. Sasa anaongoza Mradi wa Fistula katika Hospitali ya Bugando.

mwaka 1992 kushika nafasi ya kuongoza Ofisi ya Afya ya Uzazi ya shirika la Ford Foundation. Alianza kuwasiliana na watu waliokuwa wanafanya kazi ya kutatua tatizo la fistula, lakini aliona hawana ari sana na suala hili. Mwaka 1995 alihamia Mwanza ili kufanya kazi na Asasi zisizo za Kiserikali (AZISE) ambako alikutana na Dk. Gumodoka. Katika mazungumzo yao alimuuliza "Kuna cho chote kinachofanyika kuhusu fistula katika hospitali ya Bugando?" na macho yake yakafunguka. Walikaa pamoja siku ile na kuanza kushughulikia kwa makini mpango mkubwa wa matibabu ya fistula.

Kadri nilivyojifunza kuhusu athari za fistula kwa maisha ya wasichana na wanawake, ndivyo kadri nilivyohisi kuwa lilikuwa suala ambalo halipewi kipaumbele katika mitazamo yote miwili, wa afya na wa haki za binadamu. Fistula ni mfano mdogo wa masuala mengi muhimu, kuhusu maisha ya wanawake wanaoishi kwenye lindi la umaskini na wanawake wanaodharauliwa. Nikabaini kuwa kuna umuhimu wa kushughulikia tatizo hilo, lakini pia ilikuwa muhimu kuyakabili masuala mbalimbali yanayosababisha fistula, na kuweka mtazamo huu kwenye kazi ya kushughulikia tatizo la fistula.

-Maggie Bangser, mjumbe mwanzilishi wa Mradi wa Fistula katika

Hospitali ya Bugando

\section{K u we k a M s ing i}

Hatua ya kwanza ya kuanzisha mpango ilikuwa kuunda kamati ya utekelezaji, iliyoundwa na wataalamu wengine wanne waliokuwa wamejitolea kuwasaidia wasichana na wanawake wenye fistula na ambao walikuwa tayari kubeba mzigo mwingine wakati siku zao za kazi za kila siku zilikuwa zimejaa. Mbali na Maggie Bangser, wajumbe wengine katika Kamati walikuwa ni watumishi, madaktari na wauguzi toka idara ya uzazi/magonjwa ya wanawake, ganzi na 
meta 500, sio rahisi kwa wasichana na wanawake wanaosumbuliwa na ugonjwa huu wa fistula. Wajumbe wa kamati walikubaliana kimsingi kwamba, huduma za fistula zitolewe bure, kwani waathirika wengi wa ugonjwa huu ni maskini.

Mjadala uliopo sasa kati ya wanaohusika kutibu fistula, ni iwapo matibabu yatolewe bure na huduma zitengwe kwa ajili ya matunzo ya wagonjwa wa fistula nje ya hospitali, au wapate huduma hizo katika wodi hosipitalini. Wanaounga mkono hoja ya kutolewa huduma hospitalini, wanaona unyanyapaa unaweza kutokea wakati wagonjwa wa fistula wanapochanganywa na wagonjwa wengine, na jinsi wagonjwa hawa walionyimwa haki wanavyosukumwa nyuma wakati wa matibabu. Wajumbe wa kamati ya mradi waliona umuhimu wa hoja zilizotolewa, na umuhimu wa kutoa matibabu kwa wagonjwa wa fistula wodini, ili wagonjwa hao wasijisikie kuwa tofauti na wagonjwa wengine, na kwamba fistula haina tofauti na magonjwa mengine ambayo watu huhitaji matibabu hospitalini. Kamati ilijitahidi kutenga upande mmoja wa wodi ya uzazi/magonjwa ya wanawake kwa ajili ya wagonjwa wa fistula, na kulingana na maneno ya Liz Mach, "wanakuwa ni sehemu ya jamii ya fistula ndani ya jumuiya kubwa ya hospitali ya Bugando". Uchangiaji wa gharama za umeme, maji na watumishi vilikuwa pia ni vigezo vikubwa vilivyofanya mradi wa fistula uwekwe katika hospitali ya Bugando.

Miezi minane baada ya Dk. Gumodoka na Maggie Bangser kukutana, utekelezaji wa Mradi uliaanza; Dk. Gumodoka akiwa Mkurugenzi wa Mradi na Maggie akiwa Mshauri wa Mradi. Baada ya miezi minane mingine, fedha zilipatikana na ilipofikia Agosti 1997, timu ya wataalamu ilikuwa tayari kuanza mafunzo na kutoa huduma.

\section{K u j e n g a U w e z o}

Hatua ya kwanza ya Mradi ilikuwa, kutoa mafunzo kwa watumishi wa tiba, kwa kuanzia na taratibu za kurekebisha fistula. Mwezi Agosti madaktari watatu na wauguzi kutoka Hospitali ya Fistula Addis Ababa (AAFH) (angalia kisanduku) walisafiri toka Ethiopia na kuja Tanzania kuendesha Kliniki ya wiki tatu pamoja na wataalamu wa tiba kutoka Bugando na hospitali nyingine za Kanda ya Ziwa. Wataalamu toka Ethiopia walichangia utaalamu wao katika uchunguzi, upasuaji, taratibu za chumba cha upasuaji, uuguzi wa kabla na baada ya matibabu, kusimamia matukio, kuanzisha itifaki za matibabu, matumizi sahihi ya vifaa na kutoa ushauri nasaha pindi wagonjwa watokapo hospitalini. Washiriki walipata nafasi ya kuona marekebisho 38 ya matibabu ya fistula katika kipindi hicho cha wiki tatu. Kati ya wanawake 38 waliopata matibabu, wote walipona kasoro watatu, mafanikio ya asilimia 92 yaliwafurahisha wengi (kama vile Dk. Gumodoka) ambao kwa miaka mingi walijitahidi kuwasaidia wagonjwa wa fistula lakini walikosa nyenzo muhimu, maarifa ya mbinu za kisasa, na mafunzo ya uuguzi bora.

Baada ya mwanzo huu mzuri, Dk. Gumodoka na muuguzi mwandamizi wa uzazi/ magonjwa ya wanawake, walikwenda Ethiopia kwa mafunzo ya wiki nne katika AAFH. Wakiwa na nguvu mpya, walirudi Bugando na kufanya warsha kadhaa na wafanyakazi wenzao katika idara ya uzazi/magonjwa ya wanawake, ganzi na upasuaji ili kuwashirikisha kile walichojifunza. Kwa kutumia warsha hizi, mradi wa Bugando ukafuata utaratibu wa AAFH kwa muundo na wateja. Kwa mfano walianzisha mfumo mpya wa kusimamia matukio na uwekaji kumbukumbu, waliongeza ufanisi katika utaratibu wa kusafisha vitanda na mipira ya kupitishia mkojo na kuimarisha uwezo wa utoaji ushauri nasaha. Kamati ya mradi pia ilianzisha 
mfumo wa usimamizi wa uwazi wa fedha za ndani na akaunti ya fedha za kigeni, matumizi madogo madogo, na mfuko wa mahitaji muhimu ya wagonjwa. Fedha za msaada walizopata, walinunua seti kamili ya vyombo vya upasuaji wa fistula, vitanda vitatu na magodoro kumi.

Kamati ya mradi wa fistula wa Hospitali ya Bugando unashirikiana na wataalamu wawili wa upasuaji wa kurekebisha fistula na ambao kwa vipindi fulani wanatembelea Hospitali ya Bugando. Dk. John Kelly ni Mwingereza mwenye umri wa miaka 70 na ambaye amefanya matibabu ya fistula kwa zaidi ya miaka 25 katika nchi mbalimbali za Afrika na Asia. Dk. Tom Raassen, daktari mpasuaji Mholanzi anafanya kazi na AMREF na anasimamia miradi miwili ya matibabu ya fistula katika Afrika ya Mashariki. Miezi miwili baada ya wafanyakazi wa Bugando kurudi toka AAFH, Ethiopia Dk. Kelly alikuja Bugando na kuendesha kliniki kwa wiki mbili. Wanawake 35 walitibiwa wakati

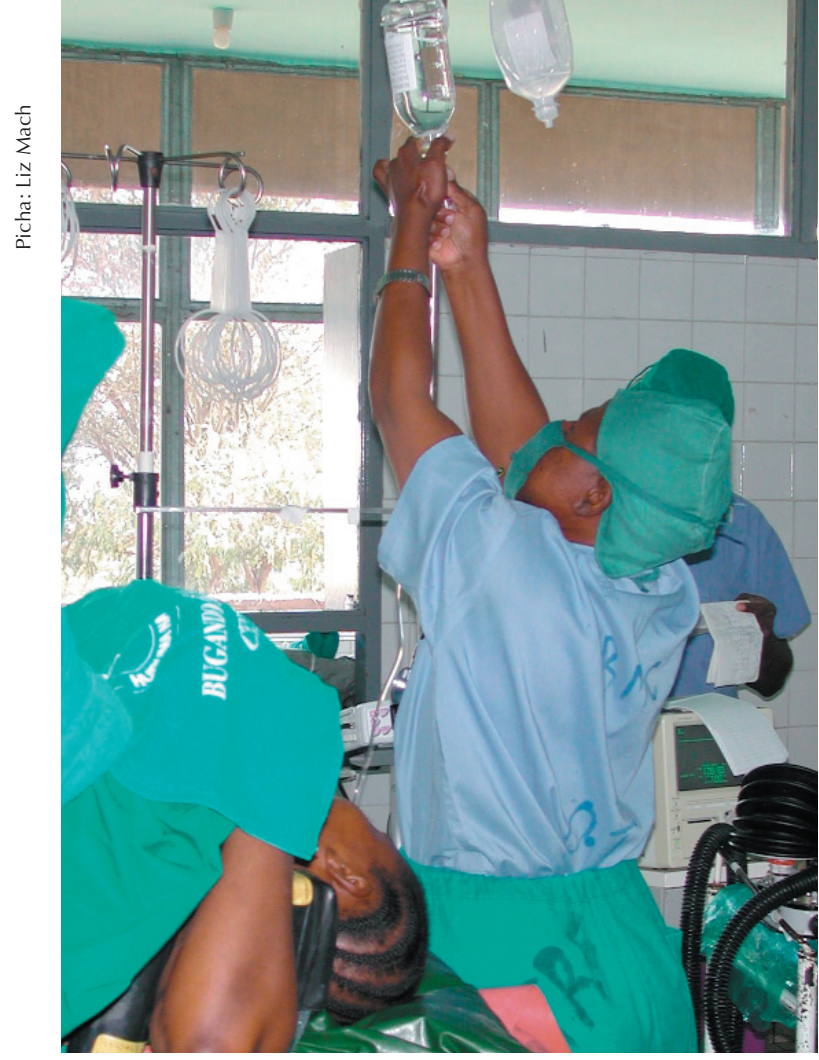

Kama sehemu ya kujenga uwezo wa kutoa matibabu ya upasuaji, wauguzi walijifunza juu ya huduma ya kabla na baada ya upasuaji, kusimamia matukio, matumizi mazuri ya vifaa na vioevu na utoaji ushauri nasaha kwa mgonjwa.

\section{Hospitali ya Fistula Addis Ababa, Et h i o p i a}

Madaktari Reginald na Catherine Hamlin, wote wakiwa wataalamu wa uzazi/magonjwa ya wanawake, waliondoka Australia mwaka 1959 kwenda kufanya kazi Ethiopia. Kwa kutambua mahitaji mengi yasiyotimizwa ya kufanya matibabu ya fistula, walianzisha Hospitali ya Fistula ya Addis Ababa. (AAFH) mwaka 1974. Tangu wakati huo AAFH imekuwa moja ya asasi kubwa za upasuaji na ufundishaji katika nchi zilizo chini ya Jangwa la Sahara katika Afrika. Hadi sasa Hospitali imetoa matibabu bila malipo kwa wasichana na wanawake zaidi ya 20,000 na kuwahudumia, kuwapa chakula, nguo na hata nauli za kurudi makwao.

Hospitali imetoa kipaumbele kwa watoa huduma kupata mafunzo. Wataalamu wa uzazi/magonjwa ya yanawake toka Ethiopia, wasiokuwa na uzoefu wa awali wa kutibu fistula, walitumia miezi miwili katika hospitali ya AAFH kama sehemu ya mafunzo yao ya uzamili, wataalamu wa upasuaji toka nchi zinazoendelea, na wataalamu wa upasuaji na uzazi/magonjwa ya wanawake wenye uzoefu, walipata mafunzo ya mwezi mmoja. Hivi karibuni AAFH ilijenga na kufungua kijiji cha furaha ("Desta Mender"), karibu na hospitali chenye uwezo wa kuhifadhi wasichana na wanawake wapatao 100 ambao wanapata matibabu ya viungo vya mwili, au wale ambao upasuaji wao haukufanikiwa. Wanawake ambao upasuaji haukufanikiwa wanatumia mifuko ya kupitishia uchafu baada ya kutobolewa tundu tumboni ambayo inahitaji kuangaliwa na wataalamu mara kwa mara, lakini wataalamu kama hao hawako katika vijiji vyao. Katika kijiji cha Furaha wasichana wanalima na kujifunza namna ya kufuma na kusuka. 
wa ziara yake, na madaktari wa upasuaji, wauguzi na wafanyakazi wa chumba cha upasuaji waliongeza na kuimarisha ujuzi wao.

Katika kipindi kifupi, timu ya Bugando iliweza kuanzisha mpango kamili wa matibabu ya fistula. Katika miezi yake 18 ya mwanzo, mradi ulitoa matibabu kwa wasichana 150 pamoja na chakula, sabuni na nauli ya kurudi makwao. Wale wagonjwa ambao hali zao za uchumi zilikuwa hoi pia walipata nguo, fedha kidogo, mahali pa kulala na chakula ili ziwasaidie wakifika nyumbani.

Ili kuelewa mahitaji na uzoefu wa wagonjwa wao, mradi ulifanya utafiti wa ubora ulioshirikisha wagonjwa 50 wa awali, wale waliofika Bugando kati ya Agosti na Oktoba 1997. Historia ya wagonjwa, angalau kikundi kidogo kilichofanyiwa utafiti, inaonyesha kuwa, walijifungua wakiwa na umri mdogo, umaskini na kutopatikana kwa urahisi kwa huduma bora za afya. Kwa mfano robo tatu kati yao walikuwa na miaka 20 au chini ya hapo walipopata mimba mara ya kwanza, chini ya asilimia 12 walisaidiwa kujifungua na muuguzi mwenye ujuzi. Wengi wa wagonjwa wa fistula walilipa dola za Kimarekani 9 kwa usafiri hadi hospitali, hiyo ni sawa na asilimia 5 ya pato la mwaka kwa kila Mtanzania.

Utafiti pia ulionyesha madhara ya fistula wanayoyapata wasichana na wanawake: Asilimia 30 ya wagonjwa wote walisema kuwa baada ya kupata fistula, waume zao walioa wanawake wengine; asilimia 90 walisema, walikuwa wanaona aibu; asilimia 67 walisema kuwa, walitengwa; na zaidi ya robo tatu walisema kuwa, kushindwa kudhibiti mkojo kulisababisha washindwe kutoka nje, kutembelea wenzao, kuwa na mahusiano ya kujamiiana, kufanya kazi au kwenda kusali (Bangser na wenzake, 1999). Matokeo ya utafiti huo yalithibitisha matarajio ya timu ya wataalamu wa fistula, pia yalitoa mwanga kwa matatizo makubwa yanayoji- tokeza, na yalivuta usikivu wa watunga sera na wafadhili.

\section{Namna Huduma Zinavyofanyakazi}

\section{$\mathrm{K}$ u i n g i a}

Wafanyakazi wanafahamu sana kuwa wanawake wanaofika hospitalini hapo wana uwezekano mkubwa wa kuwa na mfadhaiko kimwili na kimuhemko. Mara nyingi huwa wamesafiri kwa siku kadhaa au wiki kadhaa; ingawaje kimsingi Bugando inahudumia Kanda ya Ziwa, kwa kawaida wagonjwa hufika toka maeneo mengine pia, hata kutoka nchi jirani (Hivi karibuni wanawake wawili walifika baada ya safari ngumu ya siku 15 kutoka maeneo karibu na Ziwa Tanganyika iliyohusisha kusafiri kwa mashua na gari moshi.)

Safari inaweza kuwa ngumu kwa sababu kadhaa: kwa mfano wasichana wengi toka vijijini inawezekana hawajawahi kusafiri nje ya vijiji vyao, wachache zaidi wamewahi kusafiri hadi kwenye miji yenye watu wengi. Kusafiri pia ni mateso kwa mtu anayevuja mkojo. Makondakta wanasita kuwaruhusu wagonjwa wa fistula kusafiri kwenye mabasi na magari moshi, wanaweza kuwazuia wagonjwa kama wanatoa harufu mbaya au kama wasafiri wengine watalalamika. Baadhi ya wanawake wametumia miezi kadhaa kutafuta huduma kwa kuweka fedha kwa ajili ya safari na upasuaji.

Baada ya miaka miwili tangu alipopata fistula, Bahati aliamua kurudi hospitali alikojifungulia kuona kama anaweza kusaidiwa. Alikaa kwenye wodi kwa wiki moja na nusu kabla daktari kumshauri aende Hospitali ya Bugando. Akiwa bado hajatoka nje ya kijiji chake, aliogopa kusafiri mbali peke yake. Daktari wake alimweleza asiwe na hofu na alimpa mtu 
Baadhi ya wanawake huchukua miaka mingi kujadiliana ili wapate ruhusa ya kwenda kupata huduma na kukusanya fedha kwa ajili ya safari. Pia kusafiri ni mateso kwa mwanamke anayevuja mkojo. Makondakta wa mabasi na gari moshi wanaweza kuwazuia wasipande kama mwanamke anatoa harufu mbaya, au kama wasafiri wengine watalalamika. Baadhi ya wanawake hutumia miezi mingi kutafuta huduma na kutafuta pesa kwa ajili ya usafiri na upasuaji.

wa kumsindikiza. Alimlipia nauli na pia alimlipia muuguzi nauli ili amsindikize safari ya masaa 13 hadi kufika Mwanza. Bahati alipofika Bugando, alishangazwa kuwaona wasichana wengi wenye tatizo la fistula. Alikuwa hajapata kusikia kuhusu tatizo hilo na alifikiri kuwa ni yeye tu mwenye tatizo hilo. Alijisikia kufarijika kwa kuona kuwa, hakuwa peke yake na alikuwa na shauku na dukuduku kadri siku za kufanyiwa upasuaji zilivyokuwa zinakaribia. Kabla ya upasuaji, ilibidi Bahati atibiwe kwanza tatizo la upungufu wa damu na maambukizo ya kwenye uke.

\section{Kuwa unganisha}

Wagonjwa wa Fistula katika Jamii ya

\section{Hospitali}

Falsafa inayosisitizwa katika Mradi wa Fistula ni kuondoka toka hali ya kawaida ya huduma ya hospitali na kuwa na maisha ya kijamii, mahitaji ya tiba ya wasichana na wanawake yanawekwa mbele. Liz Mach, mshauri wa sasa wa mradi anasema, "Baada ya mateso ambayo wanawake wanayapitia, unyanyapaa na kutelekezwa tunataka wajisikie kuwa wanakaribishwa hospitalini. Hapa ni mahali pa usalama kwao, na wanaelewa kuwa wanaweza kuja na kupata matibabu kwa utu." Mradi ulipoanza watu waliwauliza wafanyakazi, kwa nini walikuwa wanaweka mashuka kwenye vitanda vya wagonjwa wa fistula ambapo yataharibika siku ya pili. Muuguzi wa wodi alieleza, "haidhuru kama mashuka hayo yataharibika, tutayafua. Kutandika mashuka kwenye vitanda vyao ni katika kuweka hali sawa, ni kuwarudishia utu wagonjwa hawa."

Wauguzi wana majukumu mengi katika kuwasaidia wasichana na wanawake hawa wanaoanza kupona kwa kuwapa ushauri nasaha.

Wanafahamu vizuri kuwa wagonjwa hawa wanasimanzi nyingi kwa kupoteza mtoto, mume, uwezo wa kuzaa, uwezo wa kujikimu, uwezo wa kujiamini na hadhi kijamii, na hivyo wanahitaji muda wa kuhuzunika na kuzungumza. Wakati huo huo, wauguzi pia wanawaelekeza wagonjwa wa fistula kuanza kufikiria kuhusu muda wao pale Bugando na kuhusu maisha yao ya baadaye. Wanawasaidia wagonjwa kuelezea mahitaji yao, mambo yanayowahusu, matumaini na matarajio. Wagonjwa wengine (hususani wenye umri mdogo) wanakuwa karibu sana na wauguzi na hivyo wauguzi wanakuwa kama mama zao au dada zao wakubwa. Katika juhudi zaidi za kufanya huduma kuwa ya kibinadamu na kupunguza woga, wataalamu wa ganzi mara kwa mara hukutana nao mmoja mmoja kabla ya upasuaji ili mgonjwa awe amemzoea watakapokutana chumba cha upasuaji. 


\section{Ku pata Matumaini na Kudhamiria}

Wawe wametoka karibu au mbali, wasichana na wanawake hawa kwa kawaida wana utapia mlo na hawana chochote, ila nguo walizovaa. Zaidi ya hilo, kwa kuwa wanakunywa maji kidogo ili kupunguza kiasi cha kukojoa, wengi wao wanapata ugonjwa wa usinyavushaji, kitu kinachoweza kusababisha figo kuwa na vijiwe. Mwanamke mmoja alikuwa na jiwe kwenye kibofu lenye ukubwa unaolingana na mpira wa gofu. Usinyavushaji pia unaongeza kiasi cha ammonia katika mkojo, mara nyingi husababisha ugonjwa wa ngozi au kusinyaa kwa via vya uzazi. Shughuli ya kwanza wakati wa kuingia ni kupata vipimo ili watumishi waweze kufahamu kama mgonjwa ana fistula au hutoa mkojo bila kujizuia kutokana na mfadhaiko mkubwa na iwapo ana hali nyingine mbaya au maambukizi ambayo anatakiwa kutibiwa kwanza kabla ya kufanyiwa upasuaji. Iwapo fistula imeanza hivi karibuni (ndani ya wiki moja au zaidi), watumishi wanaweza kuanza kwa kutibu mchaniko bila upasuaji (kwa kuingiza mpira wa kutolea mkojo kwa wiki nne hadi sita na kuhakikisha kuwa anapata maji ya kutosha.) Kwa kukadiria asilimia 10 ya matukio hayo yanaweza kutibiwa kwa jinsi hii.

Baada ya kuishi na fistula kwa miaka tisa, Ndebele alisikia tangazo kwenye redio kuwa matibabu yanapatikana kwa tatizo lake. Taarifa hii mpya ilimpa matumaini, lakini ilimchukua miaka miwili kuweka fedha za kutosha kuweza kufika hospitali. Raslimali za familia zilikuwa kidogo baada ya mama yake kuwa mgonjwa na kufariki mwaka 2000. Mwaka jana mjomba wake Ndebele alimchukua na kumpeleka katika mji wa karibu ambako walikaa kwa miezi mitano akijaribu kuazima fedha ili ampeleke mpwa wake hospitali. Juhudi zao zilikatishwa na familia kurudi kwenye shida tena baada ya dada yake kufariki dunia. Hatimaye Ndebele alidunduliza fedha kidogo kwa kuuza maharage na karanga, na aliazima fedha toka kwa ndugu zake kulipia usafiri kwenda hospitali ya karibu, ambako madaktari walimwekea mpira wa kutolea mkojo kwa siku 14. Tundu lake la fistula halikufunga, hata hivyo alipokuwa wodini hospitalini, Ndebele alipata taarifa kuhusu Mradi wa Fistula wa Bugando na matibabu ya bure yanayopatikana huko. Baada ya kujitahidi kwa muda mrefu kufika hospitali ya kwanza, hakuwa anajua atasafiri namna gani hadi Mwanza. Muuguzi mmoja alitambua mfadhaiko wake baada ya kusikia taabu zake, alienda kuujulisha utawala wa hospitali na aliandaliwa fedha za usafiri.

Mwaka mmoja baada ya kupata fistula, Mwasiti aliamua kwenda kutafuta msaada. Alisafiri hadi hospitali ya misheni Bunda ambako madaktari walijaribu kurekebisha tundu lake. Lakini ilishindikana na Mwasiti alikata tamaa zaidi. Wakati wa matatizo yake dada yake Mwasiti alimsaidia sana na kumfariji, pia alimwambia asiwe na wasiwasi mambo yatakuwa sawa. Siku moja walisikia matangazo redioni yaliyofafanua kuhusu fistula na yalitaja kuwa hospitali ya Bugando wanatibu fistula bure. Mwasiti aliogopa kujaribu tena, hakufikiria anaweza kutibiwa kwa sababu jaribio la kwanza lilishindikana na hakutaka kukatishwa tamaa tena. Dada yake alimtia moyo ili ajaribu tena, na muda mfupi baadaye, wote wawili walisafiri hadi Mwanza.

Baada ya kusikia matangazo ya redio kuwa Bugando wanatibu matatizo ya wanawake ya fistula, daktari wa Rhobi alimchukua katika gari na kusafiri kwa muda wa zaidi ya saa mbili katika barabara mbaya yenye mashimo na matuta kuelekea Mwanza. Rhobi alifika Bugando akisaidiwa na fimbo kumwezesha kutembea. Hakuwa na kiti kingine chochote ila shuka alilofungwa na kipande cha mpira alichokalia. Alikuwa na vipele na maambukizi ya kifua, na alikuwa anaogopa kuwa mbali na nyumbani. Wauguzi walimweleza taratibu za upasuaji, aliogopa sindano ya ganzi kwenye uti wa mgongo iliyokuwa inahitajika kuwekea ganzi, na alikuwa karibu akatae kufanyiwa upasuaji. Watumishi walitumia saa kadhaa kumtuliza na wakafanikiwa kumshawishi kwa kuomba pamoja naye na kumwambia awe na imani kuwa Mungu atamsaidia.

Hidaya, mwanamke mwenye miaka 42, ameishi na tatizo la fistula toka alipokuwa na miaka 19. Baada ya kukaa siku nne kwenye uchungu, akajifungua mtoto mfu na alianza kuvuja mkojo bila kujizuia. Mume wake alimtelekeza baada ya kupata fistula. Majaribio mawili ya kurekebisha fistula yameshindikana. Jaribio la mwaka 1980 na lile la 1988. Mwaka 2001, mfanyakazi wa afya alimweleza Hidaya kuhusu mradi wa fistula na alimshauri ajaribu tena. Alipolazwa Bugando alipimwa na akaonekana ana fistula za aina sita. Daktari aliyemfanyia upasuaji hakuwa na matumaini ya kumponyesha. 


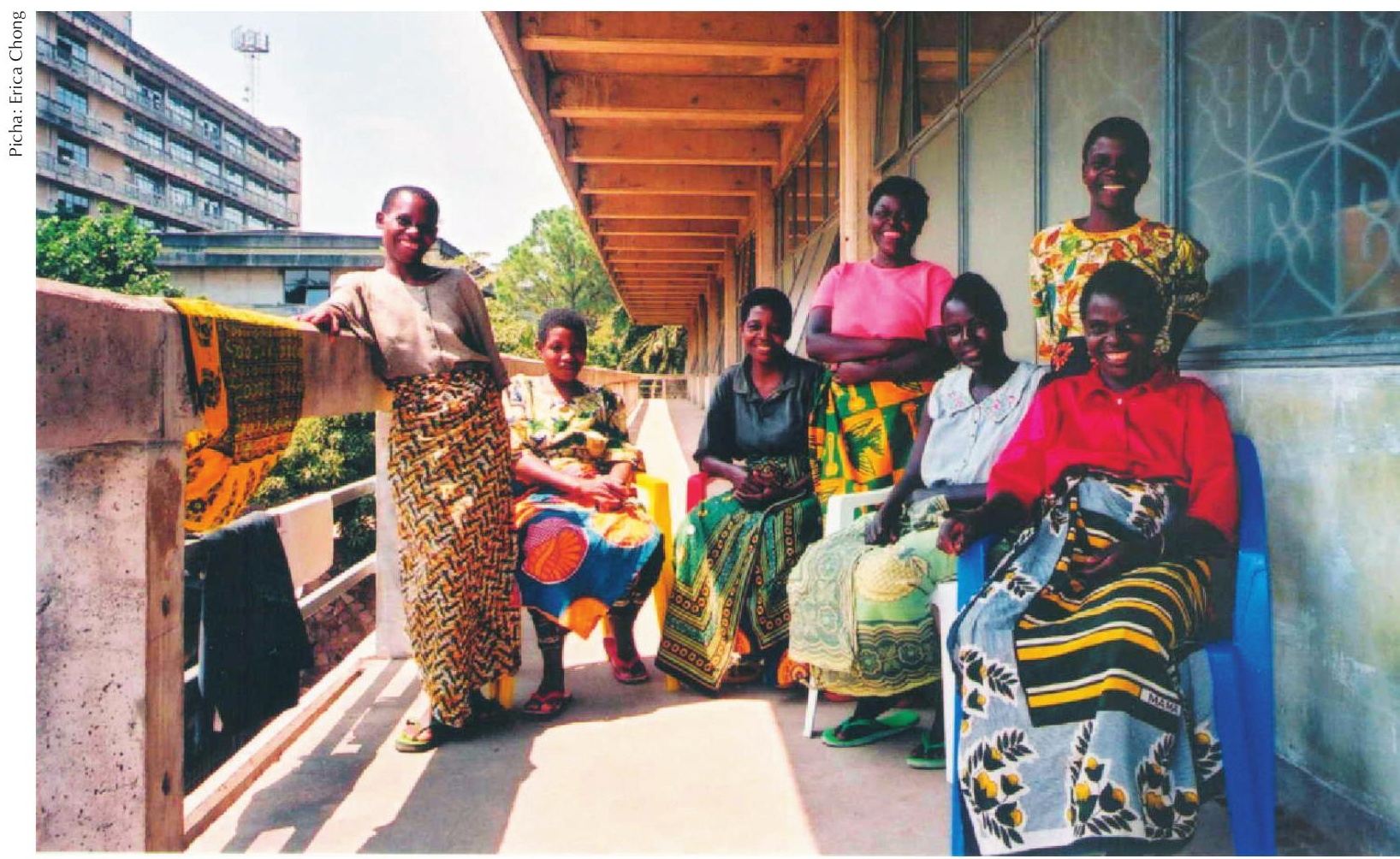

Wodi ya fistula ni mahali pa salama kwa wagonjwa wa fistula. Kwa wengi wao hii inaweza kuwa mara ya kwanza tangu kupata fistula kukubaliwa na kutibiwa kwa heshima, nafasi ya kusikitika juu ya kupoteza mtoto au kutelekezwa na mume, panatoa msaada mkubwa.

Kutegemea sababu mbalimbali, mahitaji ya mazoezi ya viungo vya mwili, matibabu ya maambukizi na magonjwa mengine kabla ya upasuaji, au upasuaji ulioshindikana, wingi wa wagonjwa, mgonjwa anaweza kukaa wodini kwa wiki kadhaa hadi miezi kadhaa. Katika muda huo, kama wauguzi au watu wanaojitolea watapatikana, wasichana na wanawake wanaweza kujifunza Kiswahili, ufumaji, ufumaji kroshia au kusoma, kuandika na kuhesabu. Liz Mach anasema, "Siyo tu miradi kama hiyo inawapa kitu cha kuangalia mbele na kitu cha kuziweka fikra zao mbali na matatizo yao, lakini pia hizi ni stadi muhimu za kurudi nazo nyumbani, na wagonjwa wanakuwa na mshikamano kwa kadri wanavyofundishana stadi hizo." Ufumaji kroshia ni shughuli inayoweza kufanywa na mtu akiwa amekaa. Jambo muhimu kulifikiria kwa wale wanaoweza kupata mfadhaiko wa kutoa mkojo bila kujizuia au wale wanaoendelea kutoa mkojo hata baada ya upasuaji. Maombi ya vifaa yaliyowekwa kwenye gazeti la 2000 la Vogue Knitting International yalipata mwitikio mkubwa. AmeriCares ilisafirisha maelfu ya ratili za nyuzi na sindano za kufumia hadi Bugando toka kwa wasomaji wa gazeti waliowaunga mkono. Ufumaji pia ulitoa nafasi nyingine kwa wodi ya wagonjwa wa fistula kujisikia wanashirikishwa kwenye jumuiya kubwa ya Hospitali ya Bugando, kwa sababu mablanketi, fulana na viatu vya watoto wanavyotengeneza hutolewa bure kwa wagonjwa wengine wanaovihitaji. 


\section{Upasuaji na Huduma B a ad a y U pasuaji}

Kutokana na mahitaji makubwa ya matibabu ya fistula, mradi unakabiliana na changamoto ya kuhakikisha kunakuwepo madaktari wa upasuaji wa kutosha. Dk. Gumodoka anafanya marekebisho yote madogo na magumu, lakini muda wake unakuwa mdogo kutokana na shughuli nyingi za utawala kama Mkurugenzi Msaidizi wa Huduma za Hospitali, Mkuu wa idara ya uzazi/magonjwa ya wanawake, na Mkurugenzi wa Mradi wa Fistula. Hivyo mradi unategemea zaidi madaktari wa upasuaji waalikwa kwa karibu nusu ya matibabu ya wagonjwa wa fistula. Dk. Kelly hukaa Bugando wiki sita kila mwaka akifanya marekebisho ya fistula karibu 120, Dk. Raassen anafanya marekebisho ya fistula 20 kila wiki mbili anazokaa hapo hospitalini.

Wakati huo huo, polepole mradi unajenga uwezo wa utaalamu wa ndani. Watanzania wawili, madaktari wa upasuaji, Dk. Marietta Mahendeka na Dk. Nhandi Ng'walida, wamemaliza mafunzo katika Hospitali ya Fistula ya Addis Ababa.

Dk. Ng'walida alieleza kuwa kuhamasika kwake kulitokea wakati wa mafunzo yake ya udakari katika hospitali kuu ya mafunzo, alipoona wagonjwa wa fistula ambao walikaa wodini kwa miezi, au hata miaka kwa sababu hakuna aliyeweza kuwatibu. Walikuwa wanamsihi awaondolee mateso yao na alijisikia vibaya kwa kuwa asingeweza kufanya chochote. Dk. Ng'walida alilalamika kutokuwa na uwezo kuhusiana na matukio ya fistula, alisema "kwa sababu wazazi wana kauli ya mwisho, wasichana wanaolewa kabla wao wenyewe hawajataka kuolewa na wanapata watoto kabla hawajawa tayari, pia katika ndoa zao hawana maamuzi, wanaume wanadhibiti fedha za familia na mwanamke anamtegemea mume katika kupata huduma za tiba."

Upasuaji wa fistula unaweza kuchukua saa moja hadi saba, upasuaji mdogo unaofanywa kuweka ganzi kwenye uti wa mgongo inahusisha kuondoa tishu ya kovu na kushona mchaniko. Mara nyingi hupandikizwa nyama nene ili kuongeza mzunguko wa damu mahali palipofanyiwa marekebisho. Upasuaji mgumu unahitaji kumpa mgonjwa dawa ya usingizi, na mirija ya mkojo kupandikizwa kwenye kibofu au kibofu cha mkojo na kipande cha utumbo mwembamba kuondolewa na kuunganishwa na mirija ya mkojo, na kumfanya mgonjwa atoe mkojo kupitia sehemu iliyopasuliwa tumboni.

Baada ya upasuaji, mgonjwa hupumzika kitandani kwa siku mbili hadi 14. (Utaratibu unatofautiana kutegemeana na daktari wa upasuaji; Kukaa

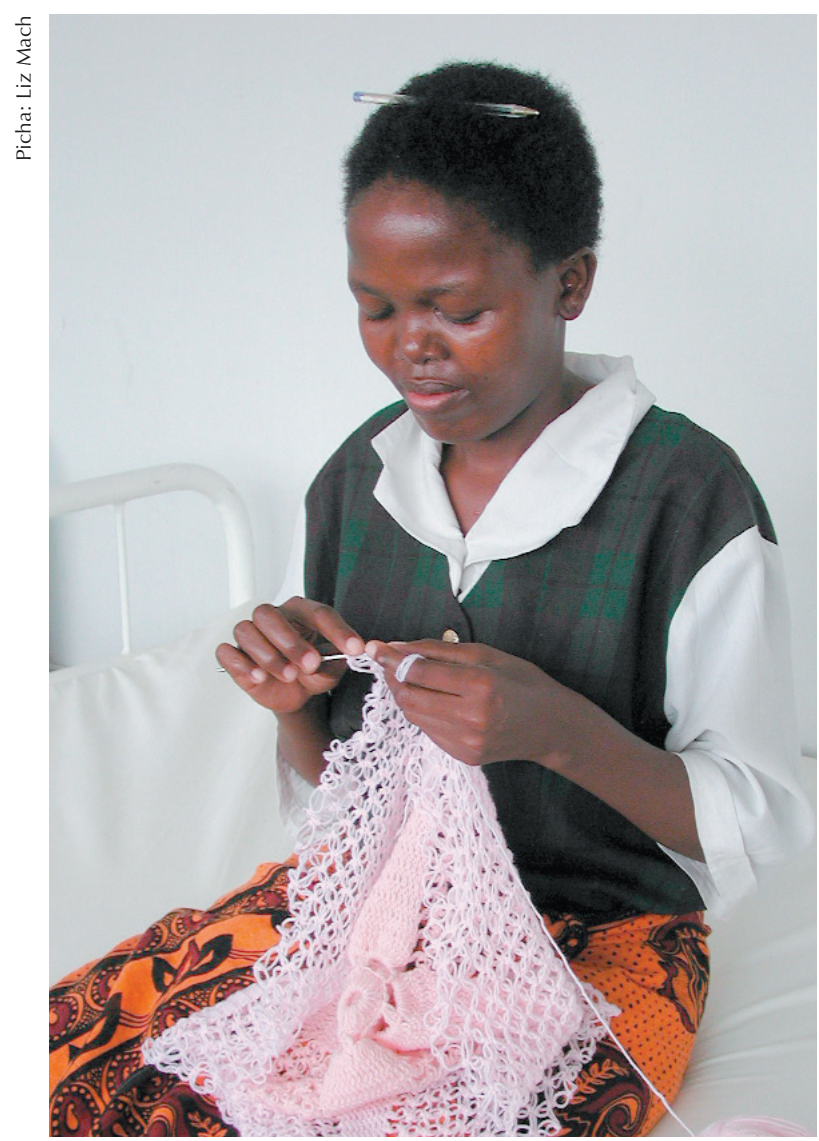

Wagonjwa wanaweza kubaki wodini kwa wiki chache au miezi kadhaa. Wanaweza kujifunza Kiswahili, ufumaji kroshia, au kusoma, kuandika na kuhesabu, shughuli zinazoweza kufanywa na mtu akiwa amekaa. Baada ya kutoa maombi ya vifaa kwenye gazeti la Vogue Knitting International, mradi ulipata maelfu ya ratili za nyuzi na sindano zilizotolewa kama msaada. 
hospitali baada ya upasuaji (ni suala linalojadiliwa na utafiti unaendelea). Ingawa upasuaji ni muhimu sana, huduma nzuri ya kuuguza ni muhimu, hususani baada ya kipindi cha upasuajii. Mara nyingi bila huduma nzuri baada ya upasuaji mahali paliposhonwa panaweza kupasuka. Kwa sababu mgonjwa bado anatumia mpira wa kutolea mkojo na ana kibofu kilichosinyaa. (Kibofu chake hakikutumika kuhifadhi mkojo tangu alipopata fistula), kupanuliwa kwa kibofu kilichoshonwa na mkojo uliobakia kunaweza kusababisha kibofu kufunguka. Pamoja na kutoa huduma na matunzo mazuri ya kidonda, wauguzi lazima waangalie kwa makini kuwa mpira wa kutolea mkojo unafanya kazi vizuri ili kuhakikisha kuwa mkojo hauzuiliwi kutoka na lazima mgonjwa apewe maji mengi na lishe bora wakati wa kutazamiwa.

Baada ya upasuaji, baadhi ya wagonjwa huendelea kupata tatizo la kutozuia mkojo kutokana na mfadhaiko, kwa mfano, kutoa mkojo bila kujizuia kutokana na kupiga chafya au kubeba vitu vizito au kutokana na kibofu kujaa wakati amelala usiku. Wauguzi wamegundua kuwa kuwafundisha kufanya mazoezi ya nyonga sakafuni inawasaidia wagonjwa wengi wenye fistula kurudisha udhibiti wa misuli yao. Liz Mach anakumbuka mahojiano kati yake na baadhi ya wagonjwa wakati wameruhusiwa kuondoka hospitali, mmoja wao alipoulizwa kama alikuwa mkavu (hajajikojolea) alijibu kuwa "Nimetoa mkojo bila kujizuia kwa sababu ya mfadhaiko. Lakini ninafanya mazoezi sasa hivi, na wewe je?" Liz alifurahi kuwa mgonjwa alikuwa ametulia pamoja na kwamba alitokwa na mkojo bila kujizuia kwa sababu aliona kuwa anaweza kudhibiti hali hiyo." Inafahamika kuwa wagonjwa wanajisikia furaha upasuaji unapofanikiwa na hawaendelei kutoa mkojo au kinyesi bila kujizuia. Katika mahojiano mengine ya kutoka hospitali mgonjwa alipoulizwa kama alikuwa mkavu, aliruka na kupandisha sketi yake na kusema "Angalia

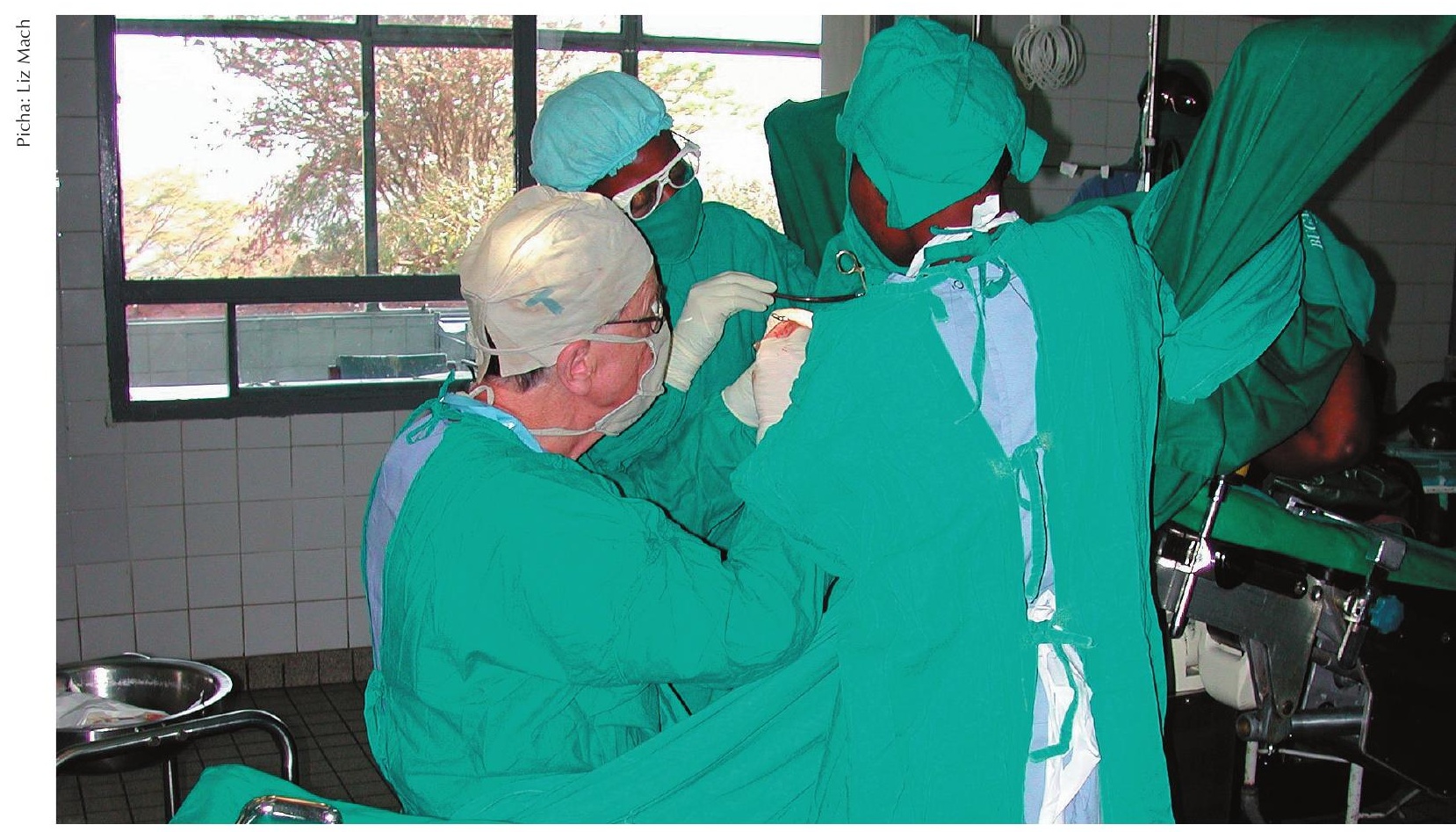

Upasuaji wa fistula unahitaji saa moja hadi saba. Pole pole Hospitali inajenga uwezo wa wataalamu wa ndani lakini bado inategemea wataalamu wa upasuaji waalikwa kufanya nusu ya upasuaji wote wa fistula. Dk. Kelly (juu) anatumia wiki sita kila mwaka katika hospitali ya Bugando. 


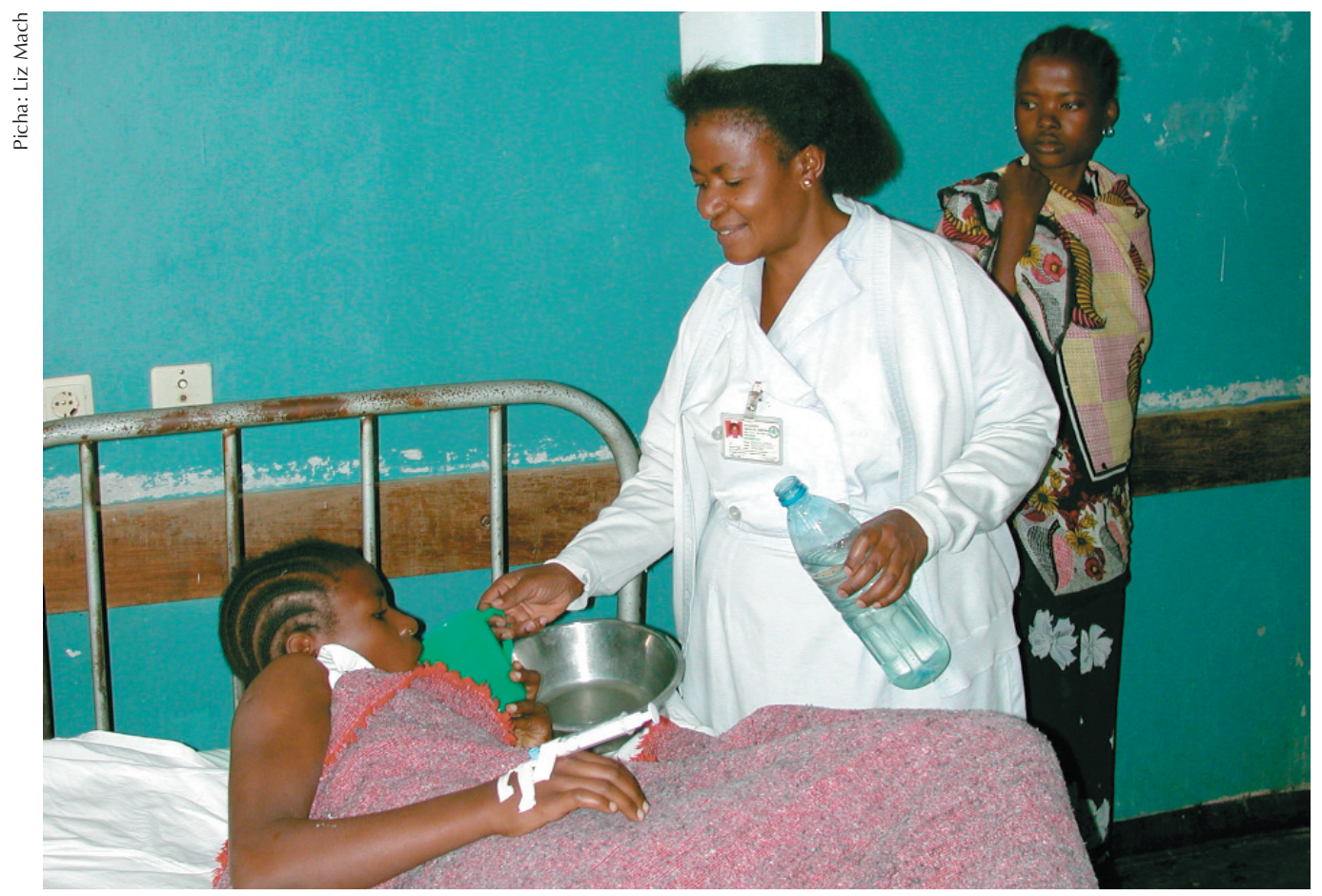

Baada ya upasuaji, mgonjwa hupumzika kitandani kwa siku mbili hadi 14. Huduma nzuri ya kuuguza baada ya upasuaji ni muhimu kwa matokeo mazuri.

nimevaa chupi kwa mara ya kwanza baada ya miaka!"

\section{Matokeo ya Upasuaji}

Mradi wa Fistula wa Bugando unachukulia wagonjwa kuwa wamepona kama watakuwa wakavu" (hii ni iwapo tu wameacha kabisa kuvuja mkojo). Uchanganuzi wa kiwango cha kupona ulijikita kwa wagonjwa 645 waliofanyiwa marekebisho ya fistula na yanaonye-

\section{Mgonjwa alipoulizwa kama}

alikuwa mkavu, aliruka juu na kupandisha sketi yake, na akasema, "tazama, nimevaa chupi kwa mara ya kwanza katika miaka mingi."

sha kuwa asilimia 74 walitibiwa na kupona, asilimia 5 hawakupona na asilimia 20 walipona kiasi (wanawake walikuwa bado na kiwango fulani cha kutoa mkojo bila kujizuia kutokana na mfadhaiko). Kutokwa na mkojo bila kujizuia kunaweza kuanzia taratibu hadi hali ile mbaya kama vile mwanamke bado ana fistula. Ingawaje katika matukio mengine kutoa mkojo bila kujizuia kunakosababishwa na mfadhaiko kunaweza kumalizwa kwa kufanya mazoezi ya nyonga sakafuni. Hali nyingine inaweza kuhitaji upasuaji zaidi na matukio machache hayatibiki. Baadhi ya matukio machache ni magumu kwa sababu mwanamke tayari amekwishapitia upasuaji usio na mafanikio mara nyingi katika hospitali ndogo, zisizo na vifaa bora. Kwa kuwa eneo lililoathirika ni dogo na kwa kuwa tishu za kovu zinajijenga kwa kila upasuaji, lakini upasuaji wa kwanza unatoa nafasi nzuri ya kurekebisha mchaniko. Liz anaelezea, "Kabla ya hapa, tulikuwa tunawaona wagonjwa wengi wakiwa wamefanyiwa upasuaji katika hospitali nyingine, upasuaji ulioshindwa, na ambao ulileta 


\section{Kuna Matarajio gani kwa Ndebele, Mwasiti, Bahati na $\mathrm{Hid}$ a y a ?}

Upasuaji wa Ndebele haukufanikiwa lakini alibakia na matumaini kuwa akijaribu mara ya pili atafanikiwa na kumaliza miaka mingi ya mateso na usumbufu.

Kitanda cha Mwasiti kinyume na matarajio yake kilikuwa kikavu siku 14 baada ya upasuaji.

Upasuaji pia ulifanikiwa kufunga fistula ya Bahati, lakini anatoa mkojo bila kujiuzia kwa sababu ya mfadhaiko.

$\mathrm{Na}$ Hidaya, mwenye umri wa miaka 42 je? Baada ya kufanyiwa marekebisho ya fistula na kitanda kuwa kikavu, watumishi wa hospitali walimlazimisha kuondoka kwa sababu alikuwa anapanda na kushuka kwenye ushoroba akiimba, kucheza na kusababisha kelele za hapa na pale.

ugumu katika kuwatibu tena." Wakati mwingine kutaja sababu dhahiri ya kushindwa kwa upasuaji ni vigumu. Labda mchaniko ulikuwa mkubwa, au tishu ilikuwa na makovu makubwa au mifumo ya kinga ya miili yao imeharibiwa na Virusi Vya Ukimwi. Watumishi wanawapa wagonjwa matunzo ya ziada na nasaha na kuwahakikishia kuwa watawasaidia na hawatatelekezwa tena.

\section{K u r u d i N y u m b a n i}

Kila mgonjwa anahojiwa kabla ya kuondoka hospitalini. Lengo moja la mahojiano haya ni kwa ajili ya kupata taarifa za kitakwimu za msingi toka wagonjwa walipolazwa, nyingi ya taarifa hizo zinahusu jamii, uchumi na viashiria afya kama umri wakati wa kuolewa, umri wakati wa mimba ya kwanza, usawa, kiwango cha elimu ya mgonjwa, huduma alizopata wakati wa mimba na uchungu, maelezo ya kina ya fistula, na hali ya maisha yake ya sasa. Zaidi ya hayo, mahojiano hayo yanatoa nafasi kutoa nasaha kwa kuwafundisha wagonjwa namna ya kujitunza wenyewe na kuwahimiza kufikiria maisha yatakuwa namna gani wanaporudi nyumbani. Baadhi ya wagonjwa hawakomi kupata hedhi; wengine wanaweza kupata hedhi baada ya upasuaji na wanaweza kupata watoto. Wasichana wanapata ushauri nasaha kuhusu uzazi wa mpango na wanashauriwa wasifanye tendo la ndoa kwa miezi 6 (kwa kupendekeza miezi 6, mshauri nasaha analenga miezi mitatu). Wanaelezwa kuwa kama wakishika mimba, wanapaswa kupata huduma kabla ya kujifungua, wajifungulie hospitali, na wawaeleze madaktari kuwa walishapata fistula. Wanashauriwa pia kuwa wanaweza kuzaa kwa njia ya upasuaji. Wauguzi wanajitahidi kuwafanya wagonjwa kuwa na mpango tayari; Wataishi wapi? Watajisaidiaje wenyewe? Anataka kuolewa tena au kama mume wake bado yupo, anafikiri mumewe atafanya nini yeye anaporudi nyumbani?

Siku kumi na nne baada ya upasuaji wake, Rhobi alikuwa mkavu na yuko tayari kurudi nyumbani. Kwa kuwa aliletwa na gari la

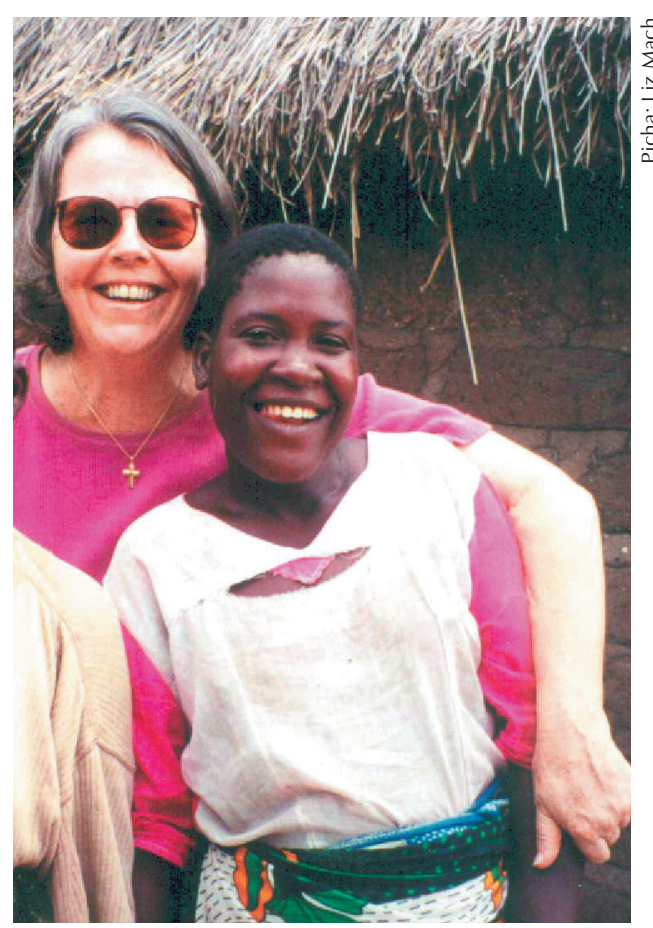

Juu, Liz Mach akiwa na mgonjwa ambaye amerudi nyumbani. 
Daktari wa Kituo cha afya na hajapata kuondoka kijijini kwao kabla ya tatizo hili, Rhobi hakujua wapi ni nyumbani kwao na jinsi gani ya kufika huko. Watumishi kadhaa waliamua kufuatilia alikopitia. Rhobi alikuwa anakumbuka kwa ujumla upande kilipo kituo cha afya kulichompelekea katika hospitali. Watumishi wa kituo walimwelekeza Rhobi na wengine waliongozana naye kuelekea kijijini kwake. Ingawa waliendesha kupitia kijijini kwake mara mbili Rhobi hakuweza kukitambua kijiji chake toka garini na hakuna kitu kilichoonekana anakijua. Mwishowe alimtambua mwanaume mmoja akiwa barabarani na akaingia kwenye gari na akawaongoza hadi kwenye nyumba ya bibi yake alikokuwa anaishi kabla ya kwenda hospitali. Rhobi aliposhuka toka kwenye gari, wanakijiji walijaa kuzunguka gari na bibi yake akatoka nje ya kibanda chake machozi yakitiririka usoni mwake, akasema "Waliniambia kuwa umefariki!"

Wagonjwa wengine hawana kitu cha kwenda nacho nyumbani. Katika matukio machache, wagonjwa hawa walifanya kazi kama wasaidizi kwenye wodi za kitengo cha fistula mpaka wakadunduliza fedha za kuwasaidia kuanzia maisha wanapoondoka kwenda nyumbani. Wasaidizi kama hao wamejenga daraja muhimu kati ya wagonjwa na watumishi wa hospitali na ni raslimali muhimu kwa kutoa taarifa. Mpango katika nchi nyingine hutumia mikakati kama hii. Tukio moja lisilo la kawaida ni la mgonjwa wa fistula aliyetibiwa katika hospitali ya AAFH alipokuwa bado kijana mdogo. Alishindwa kurudi nyumbani kwao, alisaidia upasuaji wa fistula chini ya uangalizi wa Dkt. Reginald Hamlin. Alionyesha mweleko mzuri wa kazi hii, mwishowe akawa mtaalamu wa upasuaji, hadi leo amefanya maelfu ya upasuaji na ni mmoja wa wakufunzi wazuri katika hospitali hiyo.

Ni mambo machache sana yanayofahamika kuhusu wagonjwa wa fistula wanaporudi na kujiunga na jamii zao baada ya upasuaji. Mradi wa Bugando sasa unapanga kufuatilia wasichana na wanawake waliorudi nyumbani, ili kujifunza kuhusu uzoefu wao na kama walikubaliwa kurudi kwenye jamii zao na pia kurudi kwenye ndoa zao. Je wanarudia hali zao za awali za ufukara wa kutupwa na kutokuwa na usawa kijinsia kulikosababisha hali ya fistula mara ya kwanza? Je wanaweza kupata mimba tena, kama hilo ndiyo lengo lao? Kujifungua kwa baadaye kulikuwaje? Kuna mahitaji gani wanawake wanayoyahitaji mara wanapojiunga na jamii ambayo mpango unapaswa kuyafikiria zaidi.

Je kuna mahitaji makubwa ya mafunzo ya stadi za kazi? Watumishi wanafikiria jinsi ya kushughulikia changamoto ya kuwapata wagonjwa wa zamani katika nchi zima na jinsi mahojiano yanavyoweza kufanywa vizuri zaidi ili kupata mafunzo yenye manufaa.

\section{Us imamizi wa Pamoja}

Watumishi wa mradi hufanya mikutano ya pamoja na madaktari, wataalamu wa ganzi, chumba cha upasuaji na watumishi wa utawala, mara mbili au tatu kwa mwaka. Mikutano hii kwa kawaida inachukua saa chache na inatumika kuwakaribisha wafanyakazi wapya, kuzungumzia masuala muhimu na kuandaa au kutathmini mchango wa madaktari waalikwa. Mbali na nafasi yake katika ngazi ya hospitali, kila mjumbe anahimizwa kuchangia mawazo ya kufanya kitengo kuwa na ufanisi zaidi na kuelezea mambo yanayohusika. Wajumbe wengi wa timu walisema kuwa mikutano hii ilikuwa muhimu kwa kuinua hamasa na kuhimiza utendaji. Kwa jinsi mikutano inavyofanywa kwa njia ya uwazi, inafanya kila mmoja aone kuwa mradi ni wake.

\section{Ch a n g a m o to}

Hadi mwaka 2001, karibu hospitali 50 Tanzania zinatoa matibabu ya fistula, kati ya hizo, hospitali sita tu ndiyo zinazofanya matibabu yote ya kurekebisha fistula. Hospitali ya Bugando inashughulikia karibu robo ya matukio yote (WDP 2002). Idadi ya upasuaji wa fistula inaongezeka kutoka idadi ndogo chini ya 30 kwa mwaka (Kabla ya mradi Shirikishi wa Fistula kuanza) hadi wagonjwa 228 kwa mwaka 2002, nyongeza iliyotokana na kiwango cha mafanikio kilichoboreshwa. Kukua haraka kwa mradi kulifanya kamati kuomba nafasi zaidi katika Hospitali ya Bugando. Mwaka 2002, Dk. Charles Majinge, Daktari bingwa wa upasuaji alichukua nafasi 


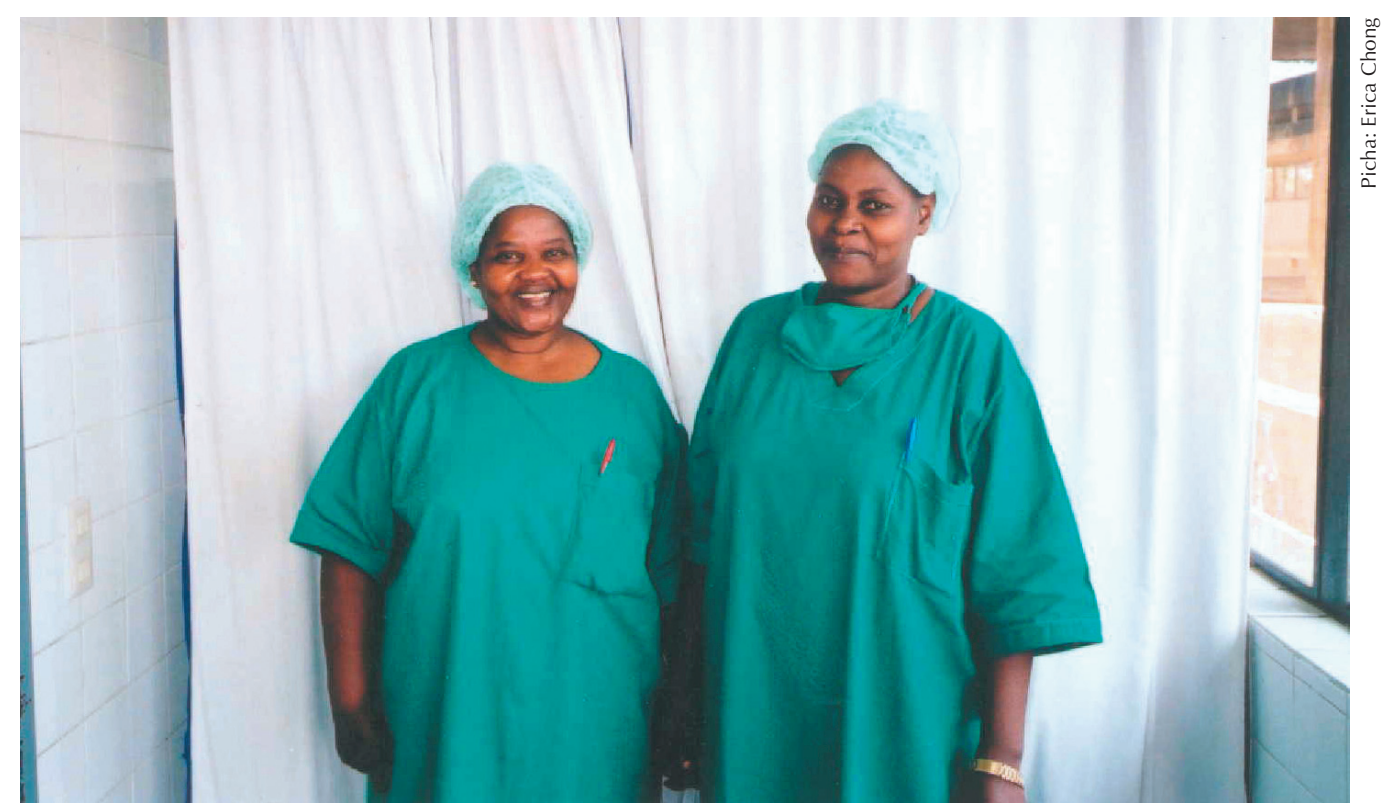

Kufanya kazi kwenye wodi ya fistula ni ngumu na ni kazi ya kujitolea. Hata hivyo mradi unavutia wauguzi wanaojituma na kuhama kumepungua.

ya Dk. Berege kama Mkurugenzi wa Hospitali, alipata mafunzo ya tiba ya fistula, alitoa wodi ya vitanda 50 vitumiwe na mradi. Hii ilipanua idadi ya vitanda vya mradi wa fistula kufikia 95. Wodi mpya inatumiwa kama mahali pa mapokezi kabla ya mgonjwa hajafanyiwa upasuaji na pia mahali pa kuponea/kupatia nafuu kama wodi kuu ikiwa imejaa. Uwezo ulipanuliwa kuruhusu mradi kuhudumia wanawake wengi zaidi, lakini pia uliongeza matatizo kuwa makubwa zaidi kuliko yalivyokuwa awali. Mengi ya matatizo hayo yanahusu upangaji watumishi na gharama za mikutano.

\section{Kutafuta Fedha za Mpango}

Watumishi wa mradi wa fistula wanakadiria kuwa kufanya upasuaji na gharama zote za vipimo vya maabara, madawa, chakula, mishahara ya watumishi na usafiri wa mgonjwa kurudi kwao hugharimu dola za Kimarekani 250 kwa kila mgonjwa. Liz Mach anajitahidi kuufanya mradi uendelee kupitia juhudi zake za utafutaji fedha. Fedha za mradi zinatokana na vyanzo vikuu vinne ambavyo ni: Maryknoll (Shirika la Kikatoliki lililopo Marekani ambako Liz atokako), Shirika la Oak Foundation, Utu Mwanamke, na mchango toka familia na marafiki wa Liz. Zaidi ya hayo AMREF inachangia dola za Kimarekani 100 (fedha kutoka Ubalozi wa Netherlands) kwa kila mgonjwa anayetibiwa na mtaalamu mwalikwa wa AMREF. Hospitali ya Bugando inatoa nyenzo (majengo, umeme, maji, matumizi ya wodi n.k.) lakini haichangii gharama za matibabu kwa wagonjwa wa fistula. Liz anasema kuwa hakuna mgonjwa aliyekataliwa kwa sababu ya ukosefu wa fedha, lakini mapambano ya kuziba gharama yanaendelea, na ufinyu wa bajeti unakwamisha baadhi ya utafiti na malengo ya mradi ya kuwafikia wagonjwa.

\section{Kuimarisha Uwezo wa Ndan i}

Kutokana na uhaba mkubwa wa madaktari nchini Tanzania, idadi ya wataalamu wa Uzazi/magonjwa ya wanawake au madaktari wa upasuaji wenye utaalamu wa kurekebisha fistula inasikitisha kuwa 
ni ndogo sana. Madaktari waalikwa wanatoa huduma kubwa inayohitajika. Dk. Kelly bado anafanya karibu asilimia 50 ya matibabu ya fistula katika Hospitali ya Bugando kila mwaka na Dk. Raassen anafanya asilimia 10 . Watumishi wa ndani mara nyingi husaidia kwenye upasuaji mgumu ili kuendeleza utaalamu wakilenga kwenye kufanya wagonjwa wao wote kupona kabisa.

Kutegemea madaktari wa upasuaji waalikwa, inakuwa ni kikwazo katika mfumo. Kwa sababu madaktari wa upasuaji wa Tanzania wanayapeleka matukio makubwa kwa madaktari waalikwa, mradi una uwezo wa kuwahudumia baadhi ya wagonjwa kwa miezi kadhaa. Zaidi ya hayo, ili huwatumia vizuri madaktari wa upasuaji waalikwa, watumishi lazima wahamasishe wiki kadhaa kabla. Wanaandaa matangazo ya redio na kupeleka barua pepe kwenye makambi ya wakimbizi na hospitali za wilaya, watumishi wa chumba cha upasuaji, wataalamu wa ganzi na wauguzi kwenye wodi, wanapitia orodha yao ya vifaa na

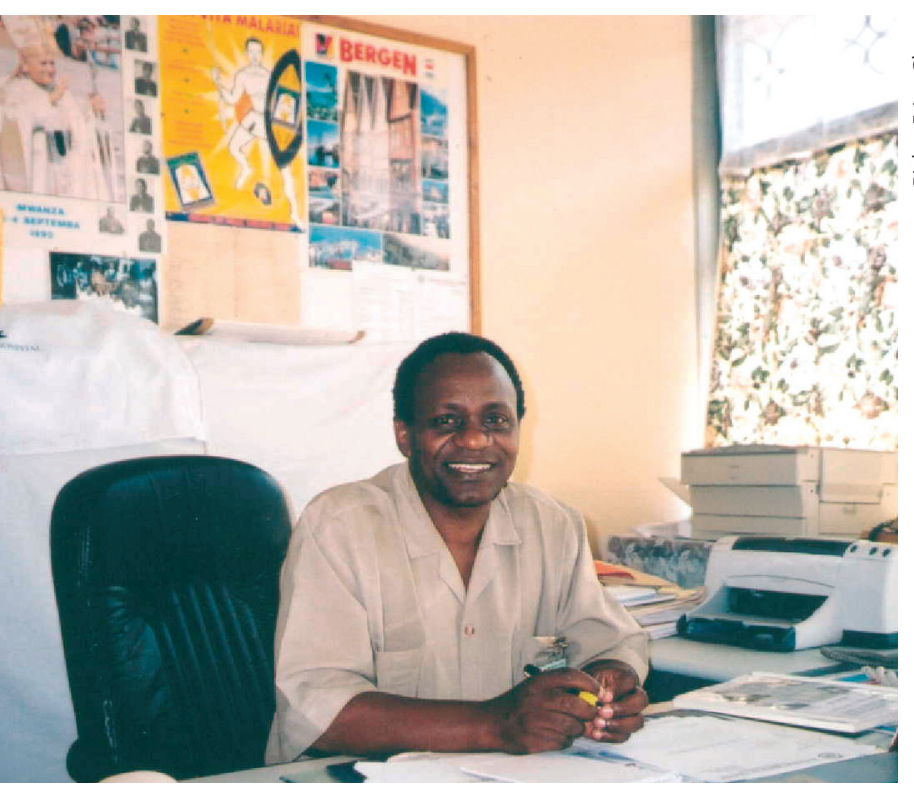

Jinsi idadi ya wagonjwa wa fistula ilivyoongezeka, Mkurugenzi wa Hospitali Dk. Charles Majinge (Daktari bingwa wa kurekebisha fistula) aliongeza idadi ya vitanda kwa ajili ya wodi ya fistula toka 50 mpaka 95. wanaagiza dawa, ganzi, mipira ya kupitishia mkojo na vifaa vingine mradi tu kuhakikisha kila kitu muhimu kinapatikana na kuandaliwa.

\section{Kuanzisha Motisha Kwa Huduma za Fistula}

Watumishi wa Tanzania hawafanyi shughuli za wagonjwa wa fistula ili kupata fedha; kwa hakika, mishahara ya serikali ni ya chini kiasi kwamba mtumishi wa hospitali anakuwa na kazi ya ziada katika sekta binafsi, kwa kuwa wagonjwa wa fistula mara nyingi ni maskini na wanahitaji upasuaji wa muda mrefu, upasuaji wa fistula hauingizi fedha nyingi. Motisha ndogo ilisababisha matatizo mawili katika Hospitali ya Bugando. Kwanza mradi ulipata tatizo la kuajiri watumishi na kupata watumishi wa kudumu, hususani madaktari. Pili madaktari walihudumia wagonjwa wengine au wenye magonjwa yanayoleta fedha zaidi kuliko wagonjwa wa fistula. Mradi uliamua kurekebisha hali hiyo kwa kupanga upasuaji wa wagonjwa wa fistula ufanyike baada ya saa za kazi za kawaida na watumishi kulipwa masaa ya ziada. Katika kiwango cha dola za Kimarekani 12 kwa siku kwa madaktari na dola 8 kwa wauguzi.

Sera ya kuwalipa watumishi wa kliniki wanaofanya upasuaji wa fistula fedha za ziada mwanzoni ilisababisha mgogoro hapo hospitali. Watumishi wa idara nyingine walishangaa nini kilichofanya wagonjwa wa fistula wawe muhimu zaidi kiasi cha kufanya huduma zao zihitaji malipo ya ziada. Kila mmoja aliyehusika kuwatunza wagonjwa wa fistula toka wale wa jikoni hadi watoa dawa waliuliza kwa nini nao wasilipwe masaa ya ziada hata kama hawakufanya kazi za ziada. Liz Mach anaamini kuwa malipo ya ziada yamesaidia kuwa na timu imara ya kliniki, pamoja na kanuni kali za hospitali. Motisha hii imechangia katika kupungua kwa rushwa na hongo, jambo ambalo linaendelea mahali ambapo 
watu wanajitahidi kupata mahitaji yao ya kila siku. Ili kutatua tatizo la kuwadumisha madaktari, katika mwelekeo wa taifa, Dk. Majinge aliamua kuruhusu madaktari wote kuwahudumia wagonjwa binafsi hospitalini hapo. Matokeo yake watumishi wengi zaidi wana mapato ya ziada na mkakati huu ulikomesha baadhi ya matatizo yaliyohusu mradi huu.

\section{Kudumisha Ubora wa}

\section{Huduma ya Uuguzi}

Kwa kuwa huduma nzuri ya uuguzi ni muhimu kwa matibabu ya fistula, mradi unasisitiza sana kuendeleza stadi za uuguzi. Juhudi hizi zimekuwa zikipata changamoto kutokana na ongezeko la wagonjwa.

Mafunzo ni muhimu ili kuwapa maelekezo watumishi wapya. (Watumishi wapya wanne waliongezeka ilipofunguliwa wodi nyingine) na kusisiti- za mbinu mwafaka. Kamati ya mradi inahimiza wataalamu kuwatembelea na kufanya warsha za papo kwa papo ambazo washiriki wanaangalia na baadaye wanafuata kanuni za uuguzi. Kamati ya mradi huandaa warsha ya vitendo ambapo washiriki huangalia na kufanya huduma. Kamati pia huandaa mafunzo ya ndani yasiyo rasmi(kuwafunza wauguzi wapya ili wapate uzoefu) hadi mfumo wa hali ya juu ulio katika muundo wa semina ya siku moja au siku mbili. Semina ya lazima kwa wauguzi wote na wauguzi wasaidizi ilifanyika mwaka 2001 kwenye wodi ya fistula na ilipokelewa vizuri. Wakufunzi waliweka matukio mawili ambapo wagonjwa walipata huduma ya kabla na baada ya upasuaji na washiriki waliulizwa waonyeshe makosa ambayo yaliwekwa kwa mahususi katika tukio. Seti nyingine ilikuwa mchezo uliojikita kwa muuguzi ambaye ni mzito, kumfuata daktari mwa-

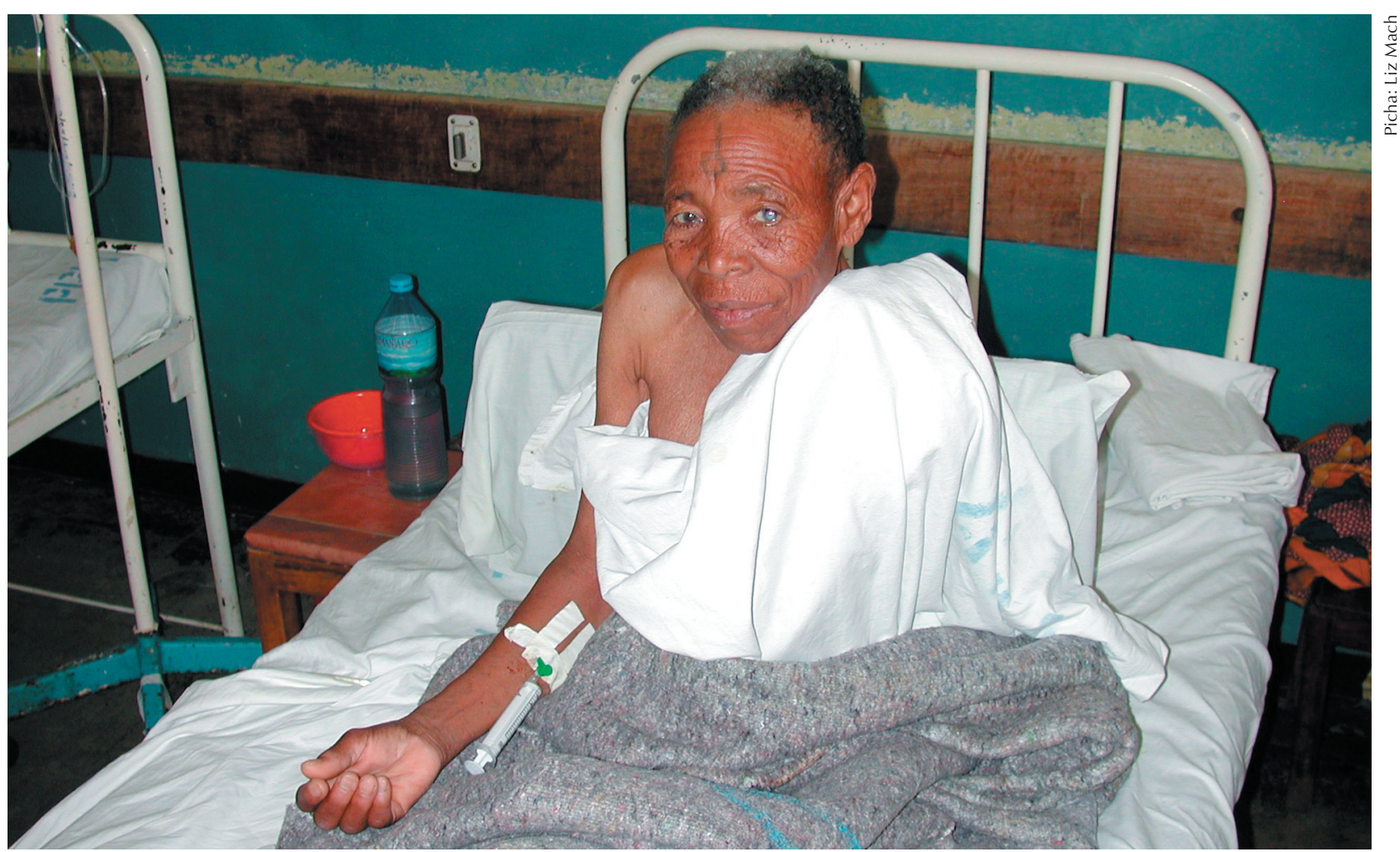

Muuguzi mkuu wa mradi anawaambia wauguzi wake "jiweke wewe mwenyewe kwenye hali ya wagonjwa wako na fikiria jinsi wanavyojisikia." 
likwa kumwelezea kuhusu matunzo ya mgonjwa au kumuuliza daktari maswali. Vitendo hivi vilifanya kuwe na majadiliano ya wazi (na kuwa na kicheko kidogo) na iliongeza nguvu za kikundi na pia kuongeza mshikamano.

Yasinta Mkama (Muuguzi Mkuu wa Wodi ya Fistula) anawafuatilia sana wauguzi wake na mara kwa mara husisitiza mazingira ambayo mradi unahitaji kuyajenga kwa ajili ya wagonjwa wao. Anakumbuka, "wauguzi wengine wanapoletwa kufanya kazi katika wodi hii, hawahudumii vizuri wagonjwa na badala yake wanatoa kauli mbaya kwa wagonjwa. Nawaambia, "ungejisikia vipi angekuwa mama yako au dada yako ana fistula, na mtu mwingine anawafanyia hivyo? "au" inaweza kuwa wewe kesho ukapata fistula. Wewe jiweke kwenye hali yao na fikiria jinsi wanavyojisikia." Kama muuguzi anajisikia vibaya kufanya kazi kwenye wodi anaweza kuomba uhamisho. Hata hivyo watumishi wa mradi wanajitahidi sana kuyaondoa matatizo kama hayo kwa kuwapa wauguzi mafunzo. Mwaka jana ni wauguzi wawili tu waliondoka toka wodi ya fistula.

\section{Nje ya Bugando: Ushirikiano wa Fistula Kitaifa}

Kufanya upasuaji wa fistula ni jambo la lazima kwa jitihada zozote za kushughulikia matibabu ya fistula, lakini mtazamo mpana wa suala hili ni kuyashughulikia mambo kama kuongeza ufahamu wa watu juu ya uwezekano wa matibabu ya fistula, kuwabainisha wanawake wenye mahitaji ya kufanyiwa upasuaji, kuanzisha mkakati wa kusimamia na kuboresha huduma, kuhamasisha fedha, ndani ya nchi na nje ya nchi; na muhimu zaidi, ni kupunguza matukio ya fistula kwa kuongeza upatikanaji wa huduma za dharura za uzazi kwa wanawake na wasichana maskini, na kuongeza umuhimu wa afya na haki za wanawake. Tanzania ina mtandao imara wa asasi zisizo za kiserikali, hospitali, idara za serikali na mashirika ya kimataifa yanayofanyia kazi masuala haya; washiriki katika mtandao huu wanabadilishana uzoefu, wanapeana taarifa na ushauri, kutoa msaada wa fedha na inategemea visa vinavyotolewa na wagonjwa vinavyogusa watu. Zaidi ya watoa huduma wa Bugando kuna watu wengine muhimu wanaosaidia.

Serikali imekuwa ni mshiriki mkuu katika jitihada za taifa za kupambana na fistula. Dk. Zackary Berege ambaye alisaidia sana hatua za awali za kuanzisha Mradi wa Fistula, kama Mkurugenzi wa hospitali, aliondoka Bugando mwaka 2000 kuwa Mkurugenzi wa Huduma za Hospitali. Wakati wa Utumishi wake katika Wizara ya Afya Dk. Berege alifanikiwa katika kuongeza ufahamu wa fistula, kutafuta fedha katika bajeti ya taifa kwa ajili ya huduma ya fistula na kupiga hatua muhimu katika uanzishaji wa mfumo wa rufaa wa taifa.

Utu Mwanamke (WDP) ilianzishwa na Maggie Bangser mwaka 2000 ili kulenga tatizo la fistula katika muktadha wa jamii mpana zaidi, ukiunganisha sera za afya na upangaji raslimali kama vikwazo kwa afya ya watu maskini. Utu Mwanamke hivi sasa ina watumishi 15 na inalenga katika maeneo ya kazi makubwa manne; (1) Utafiti wakijamii, uchanganuzi na upangaji kuhusu fistula na jamii kuwa hatarini, kwa mfano viashiria vya kijamii na kiuchumi vinavyoweza kuongeza hatari ya fistula; (2) kujenga uwezo wa taasisi katika maeneo ya tiba ya fistula na jinsia na usawa katika afya; (3) uchanganuzi wa sera na uhakiki na (4) kuhamasisha raslimali kwa ajili ya matibabu ya fistula.

Chama cha Wakunga Tanzania (TAMA) kinafanya juhudi ya kutoa elimu kwa viongozi wa vijiji, wagonjwa na wanajumuiya juu ya njia za uzazi na afya ya mama na mtoto ikiwa ni pamoja na fistula. Chama kinafanya kazi kwa karibu na Mradi wa Bugando; kwa hakika TAMA ina moja kati ya matawi yake 
Bugando na mmoja wa viongozi wake Esther Mashauri ni mjumbe mwanzilishi wa Kamati ya Mradi wa Fistula.

Namna ambavyo ushirikiano kati ya serikali, hospitali ya Bugando, jamii ya kiraia na mradi unavyofanya kazi ili kushinda mapambano dhidi ya fistula obsetrikia umeelezwa hapo chini.

\section{Mahitaji kwa Matibabu ya Fistula}

Msichana au mwanamke mwenye fistula, inawezekana haelewi nini kilimtokea au kufahamu kuwa tiba inayofaa inapatikana. Hivyo, mtandao wa asasi zinazoshughulika na fistula zimetumia njia mbalimbali kuelimisha watu kuhusu fistula na wapi mtu anaweza kwenda kupata matibabu. Katika hospitali, zahanati na vituo vya afya, wanachama wa Chama cha Wakunga Tanzania (TAMA), Kanda ya Ziwa walionyesha video, walitoa matangazo ya redio na walisaidia kuweka mabango yenye habari kuhusu fistula na kuhusu mradi wa Bugando. Watumishi wa Bugando pia wanaandaa mahojiano ya redio kwa lugha ya Kiingereza na Kiswahili na televisheni moja ilitoa kipindi cha dakika 30 juu ya mradi.

Mapema mwezi Februari 2004, Utu Mwanamke ilizindua machapisho kuhusu fistula ikiwa ni pamoja na kijitabu chao kipya kiitwacho "Sura za Utu, Mikasa Saba ya Wasichana na Wanawake wenye Fistula." Waziri wa Afya Mheshimiwa Anna Abdallah, alikuwa mgeni rasmi kwenye uzinduzi, ambapo alizungumzia mtazamo wake binafsi wa kutaka kusaidia wanawake wenye fistula. Shangazi yake alikuwa na fistula, na aliishi na hali hiyo maisha yake yote akipata mateso na kukosa raha. "Sura za Utu" vilisambazwa kupitia magazeti maarufu matatu yenye wasomaji karibu 60,000 kwa pamoja

Faida ya ushirikiano wa karibu na asasi zisizo za kiserikali zinazostawi katika Tanzania ni ya wazi. Asasi moja isiyo ya kiserikali inayofanya kazi vijijini juu ya tatizo la ukeketaji wanawake, hivi karibuni limebaini wanawake saba wenye fistula (ikiwa ni pamoja na

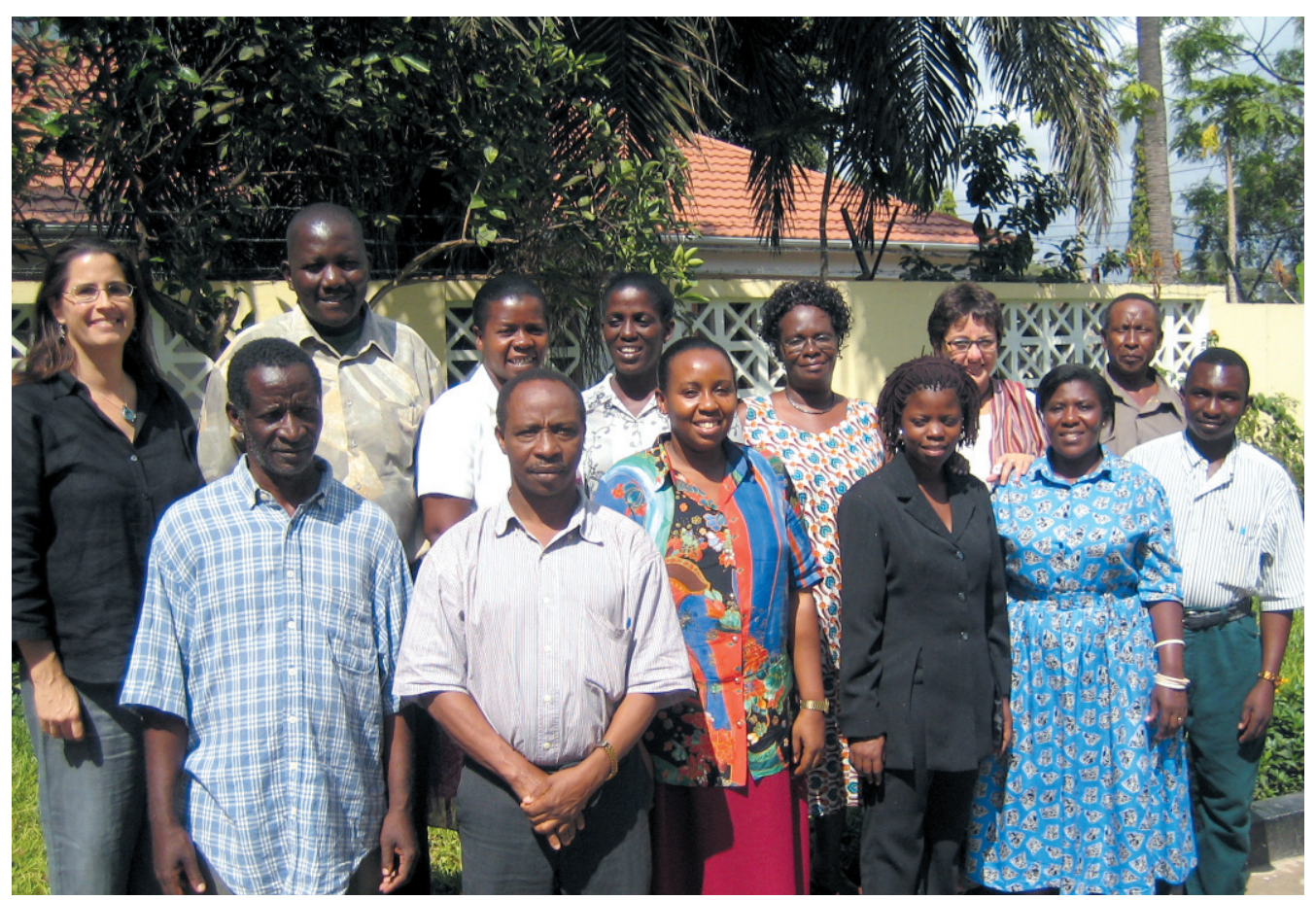

Wafanyakazi wa Utu Mwanamke 
msichana mwenye umri wa miaka 17 aliyekuwa amepigwa wakati wa uchungu uliochukua muda mrefu ili kuharakisha kujifungua). Asasi hiyo iliwasiliana na Utu Mwanamke, ambao walitayarisha usafiri na matibabu katika hospitali moja Dar es Salaam.

Idadi ya wanawake wanaohitaji matibabu ya fistula ni kubwa, kwa sababu ya matukio yaliyoachwa siyo tu kwa miaka kadhaa bali miongo kadhaa bila fistula kutibiwa. Katika kufanya utafiti kwenye vijiji vichache, Utu Mwanamke hivi karibuni imebaini wanawake 16 katika muda wa wiki mbili tu. Wengi wao wakiwa ni wazee, wakiwemo wawili walioishi na fistula kwa zaidi ya miaka 35. Mmoja baada ya mwingine, wanawake hawa waliunga 'msafara' wa watafiti uliosafiri hadi hospitali ya Bugando kwa matibabu.

\section{Kujenga Uwezo Kitaifa}

Suala la msingi kwa Mpango wa Fistula Tanzania ni uanzishaji wa mfumo wa kitaifa wa upelekaji wagonjwa katika hospital kubwa kwa matibabu. Mwaka 2001, Wizara ya Afya na Utu Mwanamke kwa pamoja walifanya utafiti mfupi katika hospitali zote za wilaya, mkoa na hospitali za misheni katika Tanzania Bara, ili kupata taarifa ni mikoa gani na hospitali zipi zinatoa matibabu ya fistula; na kujua wanawake wanazuiwa na nini kupata huduma hizo (angalia kisanduku kwa matokeo yote ya utafiti wa Fistula Tanzania).

Wanaharakati wa matibabu ya fistula, walitumia matokeo ya utafiti kuanzisha msingi wa mfumo wa kitaifa wa kupeleka wagonjwa katika hospitali kubwa kwa matibabu. Wawakilishi kutoka Wizara ya Afya, Utu mwanamke, hospitali nane, mashirika ya wafadhili na mashirika mengine, walikutana mwezi Oktoba 2002 kujadili namna mfumo wa kupeleka wagonjwa katika hospitali kubwa kwa matibabu utakavyofanya kazi, na namna utakavyoimarisha utoaji wa huduma za fistula. Walifikiria mfumo wa safu tatu, ambapo safu ya kwanza, itahusu hospi-
Asasi moja isiyo ya kiserikali inayofanya kazi katika vijiji juu ya tatizo la ukeketaji wanawake, hivi karibuni, limebaini wanawake saba wenye fistula (ikiwa ni pamoja na msichana mwenye umri wa miaka 17 aliyekuwa amepigwa wakati wa uchungu uliochukua muda mrefu ili kuharakisha kujifungua). Asasi hiyo iliwasiliana na Utu Mwanamke ambao walitayarisha usafiri na matibabu katika hospitali moja Dar es Salaam.

tali zote zinazotoa huduma za uzazi zikiwa na wahudumu wenye maarifa katika uzuiaji, uchunguzi na matibabu ya mapema ya fistula, kwa kutumia mpira wa kutolea mkojo. Safu ya pili, hospitali 8 zilichaguliwa katika maeneo yasiyopata huduma ya kutosha na zenye uwezo wa kupanua uwezo wake wa kliniki, ambazo zinaweza kufanya upasuaji mdogo wa fistula na uchunguzi, na kupeleka matukio magumu zaidi kwenye vituo vya safu ya tatu. Safu ya tatu, hospitali tano zilichaguliwa. Katika hospitali hizi madaktari na wauguzi wanaweza kushughulikia idadi kubwa ya matatizo na kumudu matukio mazito zaidi. Madaktari kutoka vituo vya safu ya tatu au madaktari wa upasuaji waalikwa wameweza kutoa mafunzo muhimu kwa madaktari wa safu ya pili. 3

Katika kuitikia yatokanayo na utafiti, kutoka kwa watoa huduma na wanawake wenye fistula, mfumo wa kupeleka wagonjwa kwenye hospitali kubwa za rufaa umerekebishwa ili kufundisha idadi kubwa ya madaktari katika hospitali za safu ya pili. Katika kuitikia juu ya vikwazo vinavyozuia wanawake wasisafiri mbali kutafuta matibabu ya fistula, wanaharakati wa matibabu waliamua 


\section{Matokeo ya Utafiti wa Fistula Tanzania}

Kati ya hospitali 172 zilizotafitiwa, 138 (asilimia 80) zilijibu, zikionyesha ari ya kiwango cha juu kuhusu tatizo la fistula. Matokeo makuu yanaonyesha yafuatayo:

1. Hospitali 50 zilifanya marekebisho ya fistula 712 kati ya mwaka 2000-01. Idadi ya marekebisho yaliyofanywa katika kila hospitali yalikuwa kati ya moja hadi 172 .

2. Hospitali kuu tano zinazofanya marekebisho ya fistula zipo katika mzingo wa nchi, hivyo matibabu hayo yanaendelea kutopatikana vijijini.

3. Ni madaktari wakitanzania 12 tu ndiyo wamepata mafunzo toka kwa wataalamu wa fistula, hivyo hospitali yoyote inayotoa huduma za fistula inategemea madaktari wapasuaji wageni wanaoalikwa kwa kufanya upasuaji.

4. Gharama za matibabu na usafiri kwenda kutibiwa ni kubwa hivyo kuwa kikwazo kwa wanawake wengi. Ingawaje hospitali nyingi zina mpango kama wa kuchangia gharama, wagonjwa wa fistula walitozwa wastani wa dola 26 za kimarekani kwa kila mgonjwa. Wagonjwa wengi wa fistula ni lazima wasafiri umbali wa zaidi ya kilometa 500 kufika kwenye sehemu moja kati ya vituo vikuu vinavyofanya upasuaji wa fistula.

5. Hospitali 19 zinazofanya upasuaji wa fistula zimedai kuwa, zina uhaba wa vifaa vya upasuaji na kuwa zana zao zimepitwa na wakati.

kuunganisha nguvu zao katika kuleta huduma karibu na wanawake wenye matatizo ya fistula kwa kuongeza uwezo zaidi na uliosambaa katika hospitali zinazoweza kufanya upasuaji mdogo .

\section{Ushawishi katika Ugawaji wa Raslimali}

Mtandao wa watu binafsi na Asasi zinazoshughulikia tatizo la fistula zinatafuta kuongeza fedha kitaifa na kimataifa kwa ajili ya matibabu. Kwa sehemu fulani, wanakusudia kuongeza fedha moja kwa moja kwenye matibabu ya fistula kwa njia ya upasuaji. Msaada na kujitolea kwa Waziri wa Afya na Katibu Mkuu wa Wizara ya Afya, Mariam Mwaffisi, kumekuwa ni muhimu katika kukusanya usikivu na kuongeza raslimali. Kutokana na jitihada zao kiasi cha dola za
Kimarekani 50,000 zilitengwa kutoka bajeti ya Wizara ya Afya mahususi kugharamia matibabu ya fistula.

Jitihada za wanaharakati ziko mbioni kuongeza raslimali kwa sekta ya afya kwa ujumla. Huu ni ushuhuda wa uwazi wa serikali na watendaji wengine. Katika jitihada hizi, Utu Mwanamke, imeombwa kushiriki katika kamati mbili muhimu, zinazotafuta mgawo zaidi kwa sekta ya afya na pia kusaidia kusimamia mikakati ya kupunguza umaskini. Ushiriki katika kamati hizi, unatoa nafasi kwa Utu Mwanamke kujenga ushirikiano na kusaidia kurekebisha maamuzi yanayoathiri moja kwa moja maisha ya watu wanaoishi katika umaskini. Zaidi ya hayo, wajumbe wa mtandao wamepigania kuliweka suala la fistula katika dondoo ya dunia kuhusu afya ya uzazi na wanashirikiana na mashirika ya kimataifa yenye ari.

${ }^{3}$ Hivi sasa watumishi wa Bugando wanasafiri kwenda hospitali zilizoko pembezoni chini ya Mpango wa AMREF wa wataalamu kufika kwa wagonjwa. Madaktari wanafanya upasuaji ,wanafundisha na kuwaelekeza watumishi wa afya wenyeji namna ya kusaidia wakati wa upasuaji, na kufanya kazi ili kuamsha ari ya madaktari wa upasuaji katika kupata mafunzo zaidi. Elizabeti Bushiri, Afisa mwangalizi muuguzi wa chumba cha upasuaji Bugando, mara kwa mara anaendesha semina ya siku moja kwa wauguzi wa upasuaji na wasaidizi wao kutoka eneo jirani. 


\section{Uzuiaji wa Fistula katika}

Tanzania

Uzuiaji wa fistula unalenga dondoo mbili zinazohusiana: Kubadilisha mwelekeo wa familia na jumuiya unaoweka thamani ndogo kwa afya ya wasichana na wanawake, na kuinua ubora wake kwa kuongeza upatikanaji wa huduma ya Mama wajawazito na huduma za dharura za uzazi.

Kwa kushirikiana na Asasi nyingine isiyo ya Kiserikali iliyopo Mwanza, Kuleana (Kituo cha kutetea Haki za Watoto), Mradi wa Fistula Bugando, mwaka 1977 uliandaa kijitabu kilichokuwa na kichwa cha habari "Haki Zangu Je?" (What about my Rights) kijitabu hiki cha lugha mbiliKiswahili/Kiingereza kinasimulia mkasa wa Eliza, msichana mdogo aliyerubuniwa na mwanaume mtu mzima kufanya naye ngono ili ampe fedha ya kulipia ada yake. Akapata mimba na baadaye akapata fistula. Kikiwa na vielelezo vya rangi angavu, kijitabu kinahitimisha kwa kusema "Watu wote pamoja na wasichana na wanawake wana haki ya kupata elimu, huduma nzuri za afya, uhuru bila vurugu, kupata chakula chenye lishe bora, kupumzika na kubarizi, kufanya maamuzi, kuheshimiwa, kuthaminiwa na kupendwa." Nakala elfu hamsini za kijitabu hiki ziligawiwa shuleni, sehemu zinazotoa huduma ya afya, makundi ya jumuiya na kwa viongozi wa dini.

Chama cha Wakunga Tanzania (TAMA) kinashughulika na uboreshaji wa matokeo ya uzazi kwa kuboresha huduma za kliniki, kuinua hadhi ya wasichana katika jamii, hususani kuepuka kuzaa mapema. Ili kusaidia kuhakikisha utalaamu wa wakunga ni wa kisasa, chama kinafanya warsha mbalimbali juu ya huduma za kabla ya kuzaa na uzazi, (hii ni pamoja na kuchunguza wagonjwa walio katika hatari ya matatizo ya mimba na matunzo ya wale wanaokuwa na uchungu wa muda mrefu).

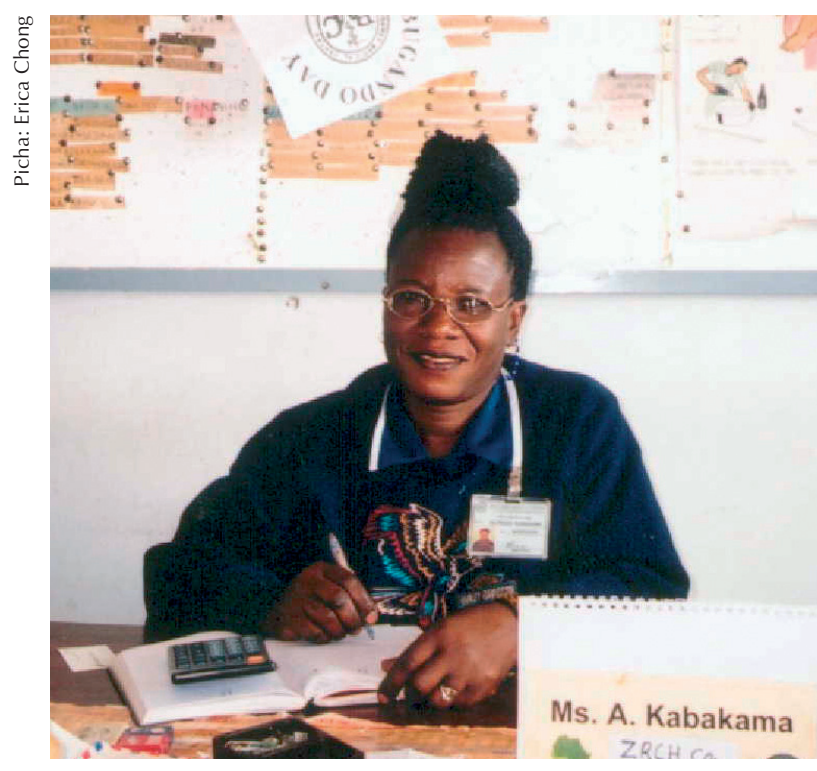

Alfreda Kabakama ni Mwenyekiti wa chama cha Wakunga Tanzania (TAMA) tawi la Hospitali ya Bugando. Lengo mojawapo la TAMA ni kutoa changamoto kwa ndoa za utotoni, na kukuza haki za wasichana na wanawake.

TAMA pia imefanikiwa kuwafikia viongozi wa dini ikiwa na ujumbe unaopinga ndoa za mapema. Wakati wa semina iliyoendeshwa na TAMA, wafanyakazi wa huduma ya afya wenyeji, waliungana na wajumbe wa jamii zao kutengeneza picha zinazotetea haki ya wasichana na wanawake kupata huduma ya afya kama sehemu ya mashindano ya mabango. Picha moja ilimwonyesha msichana anakataa kuolewa kwa sababu hajamaliza shule; picha nyinginge ilionyesha mwanaume akimkimbiza mama mjamzito hospitali kwa ajili ya huduma ya uzazi. Mradi wa Bugando ulizitumia baadhi ya picha hizo kwenye kalenda ya mwaka 1999, na pia ilimpa kazi msanii mmoja kuzikuza kuwa kama picha za ukutani ili zionyeshwe katika jamii zote zilizoshiriki. Watumishi wa Bugando wana matumaini kuwa picha hizo zitatundikwa kwenye maeneo ya umma kama vile sokoni, na hospitali za wilaya na hivyo zitachokoza majadiliano kuhusu kwanini wasichana na wanawake wana uzoefu mbaya juu ya matibabu ya afya ya uzazi. 
Kwa kushirikiana na washiriki wanne wenyeji, pamoja na EngenderHealth, Utu Mwanamke inafanya utafiti zaidi ili kuonyesha mwanga katika 'viashiria vya fistula.' Mahojiano, majadiliano ya vikundi lengwa, na mbinu shirikishi za utafiti zilizo wahusisha wasichana na wanawake, pia wanafamilia, wanajamii na watoa huduma zinatumika kuweka kumbukumbu ya jinsi nguvu za kijamii na kiuchumi zinavyoweza kuwaweka wasichana na wanawake katika hatari; wakati wa uchungu, kujifungua na baada ya kujifungua, kwa kuzingatia kama

\section{Gharama za Matibabu ya fistula} ni ndogo sana kuliko hasara inayopatikana na mwanamke anapotekelezwa na jamii kwa maisha yake yote. Zaidi ya hayo, kuanzisha mpango wa fistula siyo gharama sana.

wanaweza kupata huduma ya afya. Utafiti unayaweka bayana mambo kama fursa ya kuondoka nyumbani, utaratibu wa kuwatibu watu maskini mwishoni, kutokuwepo na usafiri wa dharura katika jamii; na kutokuwa na ufahamu kati ya watoa maamuzi katika familia (hususani waume na mama wakwe) juu ya ucheleweshaji wa ndoa na kuzaa mapema na kutafuta huduma ya dharura ya tiba wakati wa uchungu wa muda mrefu. Matokeo ya utafiti yatatumika kuanzisha njia ifaayo mahali pale kuzuia na kumudu fistula katika nchi nzima ya Tanzania kwa kushirikiana na jumuiya ya watoa huduma za afya. Katika utafiti wa pili, Utu Mwanamke inachunguza uzoefu mbaya wa wanawake maskini kuhusu huduma ya afya, pamoja na athari za sera ya uchangiaji, vizuizi katika kupata huduma na ubora wa huduma kwa jumla.

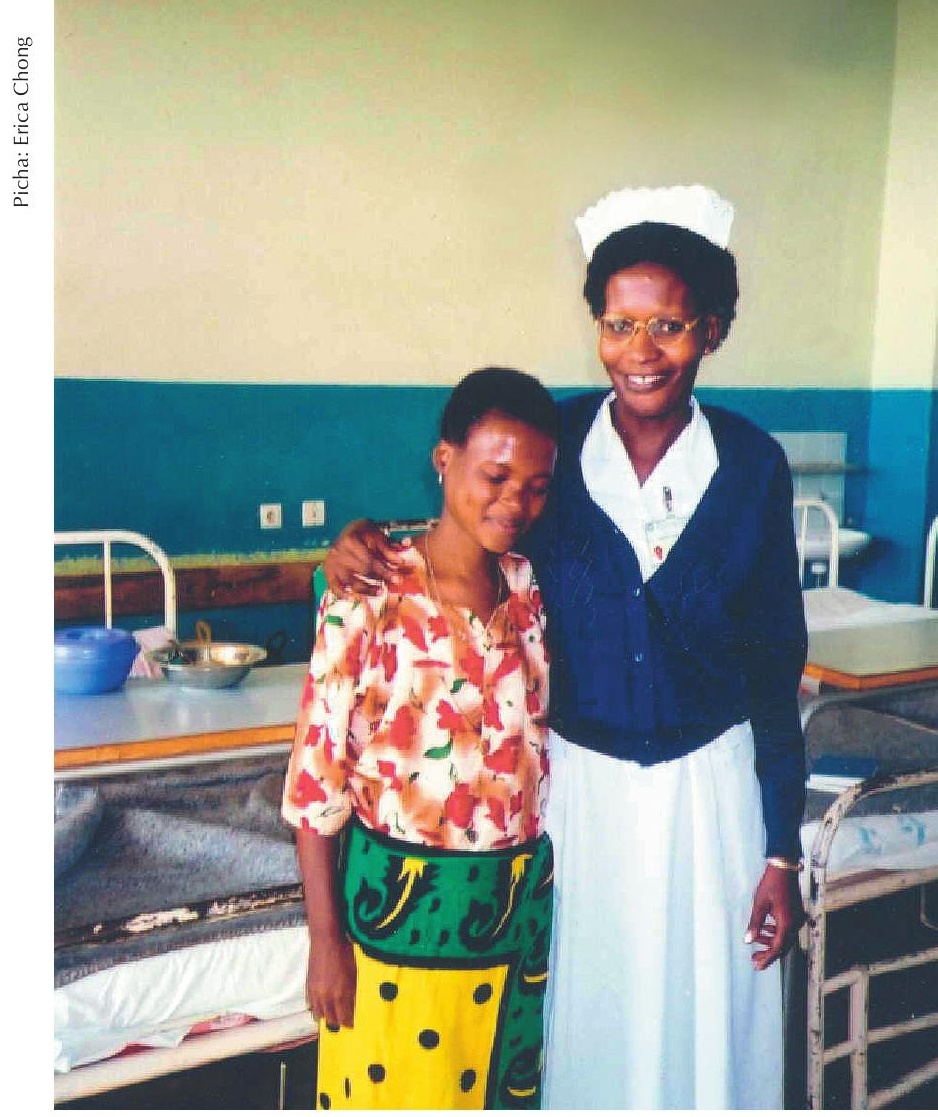

Kukiwa na watumishi wa upasuaji na zana za kutosha, kunaweza kurekebisha idadi kubwa ya fistula na kuleta mafanikio kwa haraka.

\section{TUMEJIFUNZA NINI}

Wahusika wengi, watoa huduma na watunga sera,wakielewa kwamba fistula ni matokeo ya si tu kutokuwepo na huduma ya dharura ya uzazi bali pia umaskini na kutokuwa na usawa wa kijinsiawameonyesha kukata tamaa katika kutoa matibabu ya fistula kama kipaumbele katika huduma ya afya. Jitihada za kuzuia fistula zinaweza kuonekana zimeelemewa. Uzoefu wa mtandao wa fistula katika Tanzania unathibitisha kuwa ushirikiano wa hospitali, serikali na asasi mbalimbali unaweza kuchukua jitihada za aina mbalimbali zikihusisha uchumi, jamii na ufundi. Somo kutokana na uzoefu huu linatoa mfano wenye kuleta matumaini ya kupambana na tatizo la fistula ulimwenguni. 
Mtazamo wa kushirikisha nyanja nyingi unahitajika ili kupambana na fistula kwa ufanisi. Hata ile mipango inayoanza na msisitizo finyu wa matibabu kwa njia ya upasuaji (kuliko kuzuia) mara tu hupambana na matatizo ya kijamii na kiuchumi (kwa mfano upungufu wa madaktari wa upasuaji, athari hasi juu ya hospitali na wodi za upasuaji, mahitaji ya kutoa ushauri nasaha na msaada kwa wagonjwa, ukosefu wa uelewa wa umma juu ya tatizo hili. Mahitaji ya wanawake hayapewi kipaumbele, matatizo ya usafiri, changamoto za kuungana tena na familia au jamii na tatizo la fedha.

Ingawaje matibabu ya fistula hayana kiwango cha mafanikio kwa asilimia 100, matukio mengi yanaweza kutibiwa kwa mafanikio au kupata nafuu sana kama upasuaji ni wa ubora wa kiwango cha juu. Tofauti inayopatikana kwa upasuaji uliofanikiwa kwa maisha ya mwanamke ni kubwa sana.

Watunga sera, madaktari wa upasuaji na wafadhili ambao wameona fistula kuwa ni tatizo kubwa lisilobadilika mara nyingi wanatiwa moyo na simulizi binafsi na hii huwahamasisha kusaidia kupambana na tatizo la fistula kwa kutoa raslimali zao.

Matibabu ya fistula ni uwekezaji mzuri kiuchumi. Gharama yake dola za Kimarekani 250, ni ndogo sana kuliko madhara yanayotokea wakati mwanamke anapotelekezwa na jamii maisha yake yote. Zaidi ya hapo kuanzisha mpango wa fistula sio gharama kubwa.

Matukio yaliyoenea ya fistula yanaashiria matatizo yanayowakabili wanawake maskini kwa ujumla; Jitihada za kupunguza matukio ya fistula zinaweza kusaidia kujenga ufahamu wa haki za wanawake katika huduma ya afya na masuala ya uwezo wa kijinsia.

\section{Mar e j e o}

Bangser, Maggie et. al. 1999. "A comprehensive approach to vesico-vaginal fistula: A project in Mwanza, Tanzania." In Safe Motherhood Initiatives: Critical Issues, Eds. Marge Berer and T.K. Sundari Ravindran. Oxford: Blackwell Science. Pp. 157-165.

Mpangile, Gottlieb S. et. al. 1993. "Factors associated with induced abortion in public hospitals in Dar es Salaam, Tanzania." Reproductive Health Matters 2: 21-31.

National Bureau of Statistics of Tanzania (NBS) and Macro International (MI). 2000. Tanzania Reproductive and Child Health Survey 1999. Dar es Salaam and Calverton, MD: NBS and MI.

Population Reference Bureau (PRB). 2000. The World's Youth 2000. Washington, DC: PRB.

$$
\text { 2001. Youth in Sub-Saharan }
$$

Africa: A Chartbook on Sexual Experience and Reproductive Health. Washington, DC: PRB. . 2002. 2002 World Population

Data Sheet. Washington, DC: PRB.

The POLICY Project (TPP). Maternal and Neonatal Program Effort Index: Tanzania.<http://www.policyproject.com/p ubs/MNPI/Tanzania_MNPI.pdf.>Accessed 11 Jul 2003.

United Nations Development Programme (UNDP). 2003. Human Development Report 2003. New York: Oxford University Press.

UNFPA (United Nations Population Fund). 2003. The Second Meeting of the Working Group for the Prevention and Treatment of Obstetric Fistula. New York: UNFPA.

Women's Dignity Project (WDP). 2002. Tanzania Fistula Survey 2001. Dar es Salaam: WDP.

World Bank. 2003. Country Data: Tanzania at a Glance. <http//:www. worldbank. org/data/countrydata/countrydata.html>. Accessed 11 July 2003. 


\section{Kuhusu Waandishi}

Thoraya Ahmed Obaid ni Mkurugenzi Mtendaji wa UNFPA, United Nations Population Fund, chanzo muhimu cha msaada wa idadi ya watu. UNFPA inaongoza Kampeni ya Kumaliza Fistula duniani.

Erica Chong ni msaidizi katika Kitengo cha Mipango ya Kimataifa Cha Population Council New York.

\section{Uzalish a ji}

Mhariri: Debbie Rogow

\section{Msaidizi wa Utafiti na Uhariri:}

Michelle Skaer

\section{Mhariri wa Mradi:}

Karen Tweedy-Holmes

Msanifu: Mike Vosika

Tafsiri: Paul Constance (Kisipaniola) na Jeannette Ndong (Kifaransa)

\section{Was h a r i}

Delia Barcelona Laura Laski

Gary Barker

Ann Leonard

Carmen Barroso

Judith Bruce

Ann McCauley

Liz McGrory

Batya Elul

Manisha Mehta

Susan Galdos

Suellen Miller

Kirrin Gill

Saumya Ramarao

Francoise Girard Julie Reich

Nicole Haberland Ann Starrs

Katherine Kurz

Cynthia Steele

\section{S h u k r a n i}

Erica Chong anawashukuru wafanyakazi wenye moyo wa kujitolea wa Hospitali ya Bugando, hususani Elizabeth Mach na Yasinta Mkama kwa kutoa taarifa muhimu na kuwezesha ukusanyaji wa takwimu. Zaidi anawashukuru wasichana na wanawake ambao walitoa maoni na uzoefu wao kwa uwazi kuhusu fistula.

Utu Mwanamke inawashukuru Festa Andrew, Alice Nkhoma - Wamunza, Eliezer K.F. Chiduo na Jamillah Mwanjisi kwa msaada wao wa kutafsiri jarida hili katika lugha ya Kiswahili. 
Tunakaribisha maoni yako juu ya jarida la Quality/Calidad/Quality. Ukipenda utumiwe matoleo ya jarida hili, tuandikie kupitia barua pepe: qcq@popcouncil.org. Mengi ya Matoleo yaliyopita yanapatikana kwenye mtandao <www.popcouncil.org/publications/qcq/default.htm>. Nakala zifuatazo zinapatikana, unaweza kuagiza moja au zaidi kupitia barua pepe:

Celebrating Mother and Child on the Fortieth Day: The Sfax Tunisia Postpartum Program (English only), no. 1, 1989.

Man/Hombre/Homme: Meeting Male Re $\neg$ productive Health Care Needs in Latin America (English, Spanish), no. 2, 1990.

The Bangladesh Women's Health Coalition (English only), no. 3, 1991.

By and For Women: Involving Women in the Development of Reproductive Health Care Materials (English, Spanish), no. 4, 1992.

Gente Joven/Young People: A Dialogue on Sexuality with Adolescents in Mexico (Engᄀlish, Spanish), no. 5, 1993.

The Coletivo: A Feminist Sexuality and Health Collective in Brazil (English, Portuनguese, Spanish), no. 6, 1995.

Doing More with Less: The Marie Stopes Clin $\neg$ ics of Sierra Leone (English only), no. 7, 1995.

Introducing Sexuality within Family Plan $n$ ning: Three Positive Experiences from Latin
America and the Caribbean (English, Spanᄀish), no. 8, 1997.

Using COPE to Improve Quality of Care: The Experience of the Family Planning Associ-ation of Kenya (English, Spanish), no. 9, 1998.

Alone You Are Nobody, Together We Float: The Manuela Ramos Movement (English, Spanish), no. 10, 2000.

From Patna to Paris: Providing Safe and HuᄀmaneAbortion (English only), no. 11, 2001.

Universal Sexuality Education in Mongolia: Educating Today to Protect Tomorrow (En?glish only), no. 12, 2002.

What about Us? Bringing Infertility into Reproductive Health Care (English only), no. 13, 2002.

"My Father Didn't Think This Way": Niger-ian Boys Contemplate Gender Equality (Enᄀglish, French), no. 14, 2003.

Linking Reproductive Health to Social Pow-er: Community Health Workers in Belize and Pakistan (English only), no. 15, 2004. 


\section{U t u M w a n a m k e}

Women's Dignity Project

Utu Mwanamke ilianzishwa kwa nia ya kuzuia na kudhibiti tatizo la fistula, ili kuinua hadhi na haki za wanawake wanaoishi na fistula, na kuendeleza masuala ya afya na jinsia na uwiano wa kupata huduma za afya. Msingi wa kazi yetu ni ufahamu kuwa hali ya afya inayowakabili watu maskini ni matokeo ya sababu za kijamii, kiuchumi na kisiasa ambazo husababisha umaskini.

Utu Mwanamke inalenga:

- $\quad$ Kuelewa vizuri zaidi athari ziwapatazo wasichana na wanawake wanaoishi na fistula

- $\quad$ Kuiwezesha jamii na mashirika kuchukua hatua maridhawa juu ya tatizo la fistulana mapungufu yanayowakabili watu maskini

- $\quad$ Kuhamasisha midahalo ya umma juu ya kudhibiti mapungufu hayo

- Kushawishi mipango na sera za kukuza utu na haki za watu maskini Utu Mwanamke pia inawasaidia wasichana na wanawake kupata matibabu ya fistula na kuwawezesha kuanza kuishi maisha mapya. Kama ungependa kumsaidia msichana au mwanamke mwenye fistula au kazi inayofanywa na Utu Mwanamke tafadhali wasiliana nasi. 

Q/C/Q Toleo la 16

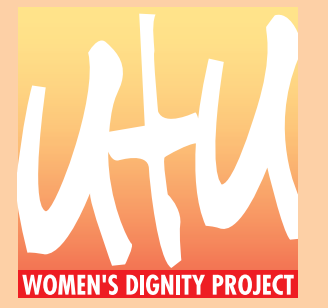

Quality/Calidad/Qualité/ 